INFNFE-20-96

\title{
Helioseismology and standard solar models
}

\author{
S. Degl'Innocenti ${ }^{1,2}$, W. A. Dziembowski ${ }^{3}$, G. Fiorentini ${ }^{1,2}$, and B. Ricci ${ }^{2}$ \\ ${ }^{1}$ Dipartimento di Fisica dell'Università di Ferrara, via Paradiso 12, I-44100 Ferrara, Italy \\ ${ }^{2}$ Istituto Nazionale di Fisica Nucleare, Sezione di Ferrara, via Paradiso 12, I-44100 Ferrara, Italy \\ ${ }^{3}$ Copernicus Astronomical Center, ul. Bartycka 18, 00716 Warsaw, Poland
}

(November 1996)

\begin{abstract}
We present a systematical analysis of uncertainties in the helioseismological determination of quantities characterizing the solar structure. We discuss the effect of errors on the measured frequencies, the residual solar model dependence and the uncertainties of the inversion method. We find $\mathrm{Y}_{p h}=$ $0.238-0.259, R_{b} / R_{\odot}=0.708-0.714$ and $\rho_{b}=(0.185-0.199) \mathrm{gr} / \mathrm{cm}^{3}$ (the index $b$ refers to the bottom of the convective envelope). In the interval $0.2<R / R_{\odot}<0.65$, the quantity $U=P / \rho$ is determined with and accuracy of $\pm 5^{\circ} \%$ or better. The predictions of a few recent solar model calculations are compared with helioseismological results.
\end{abstract}

\section{INTRODUCTION}

A Standard Solar Model (SSM) may be defined as a description of the solar interior which reproduces the observed properties of the sun within observational errors, by adopting a set of physical and chemical inputs chosen within the range of their uncertainties.

From the point of view of stellar evolution, the sun is a well known structure. Confidence in SSMs rests on the success of stellar evolution theory to describe many, and more complex, evolutionary phases in good agreement with observational data. Before the advent of helioseismology, however, a solar model had three (essentially) free parameters - the initial helium and heavier elements abundances, $\mathrm{Y}$ and $\mathrm{Z}$, and the mixing length parameter $\alpha-$ for producing three observables: the present radius, luminosity and heavy elements content of the photosphere. From this point of view, the success of SSMs to reproduce these observables may look as a not too big accomplishment.

The solar neutrino puzzle (see e.g. [1]), i.e. the disagreement between the SSM predictions and the results of the four solar neutrino experiments [4], originated many discussions about the validity of the SSMs and prompted many attempts to build alternative solar models, none however capable of solving the discrepancy.

In recent years helioseismology has added important information on the solar structure. A SSM has now to account for these additional data. 
Helioseismology (see [8] for an introduction and [9,10] for extensive reviews) provides severe constraints and tests of solar model calculations. For instance, from inversion (deconvolution) of helioseismological data one can infer the photospheric helium abundance $\mathrm{Y}_{p h}$ [11,12] and the depth of the convective zone $R_{b}$ [13]. The helium mass fraction, which was used as an essentially free parameter to match the observed solar luminosity, is now strongly constrained. Still one can adjust $\alpha$ in order to get the proper solar radius, $R_{\odot}$; however there is no parameter which can be tuned to get $R_{b}$. The comparison between the theoretically predicted value of $R_{b}$ and the value inferrred from helioseismology is a test of the model.

Helioseismology can tell a lot about the solar interior, however, it cannot replace SSMs. As an example, helioseismology determines the sound speed down to the solar core to high accuracy, but it cannot give the temperature profile in the energy production region. In order to calculate the several neutrino luminosities (pp, ${ }^{7} \mathrm{Be}, \mathrm{CNO},{ }^{8} \mathrm{~B} \ldots$ ), which crucially depend on this profile, SSMs are needed.

For people interested in solar neutrinos, the basic question is thus:

- Which solar models - if any - are acceptable, i.e. consistent with helioseismology?

Before addressing this question, one has to provide a quantitative answer to the following one:

- How accurate are the helioseimological determinations of quantities characterizing the solar interior?

The present paper is essentially devoted to this issue: we perform a systematic and possibly exhaustive investigation of the uncertainties of the helioseismological approach, in order to estimate the global error to be assigned to helioseismological determinations of the solar properties. With this spirit, we analyse the helioseismological determinations of several physical quantities $Q$ characterizing the solar structure. Concerning the outer part of the sun, we discuss $\mathrm{Y}_{p h}, R_{b}$, the sound speed $c_{b}$ and density $\rho_{b}$ at the bottom of the convective zone. Then we consider the "intermediate" solar interior $\left(x=R / R_{\odot}=0.2-0.65\right)$, analysing the behaviour of the squared isothermal sound speed, $U=P / \rho$. Finally we investigate the inner region $(x \leq 0.2)$, where nuclear energy and neutrinos are produced.

For each quantity $Q$ we determine the partial errors corresponding to each uncertainties of the helioseismological method. This approach - which will be better elucidated in sections 【I and III - is needed since helioseismology measures only the frequencies $\{\Omega\}$ of solar pmodes, and quantities characterizing the solar structure are indirectly inferred from the $\{\Omega\}$ 's, through an inversion method. Schematically, the procedure is the following:

a) One starts with a solar model, giving values $Q_{\text {mod }}$ and predicting a set $\left\{\Omega_{\text {mod }}\right\}$ of frequencies. These will be somehow different from the measured frequencies, $\Omega_{\odot} \pm \Delta \Omega_{\odot}$

b) One then searches for the corrections $q$ to the solar model which are needed in order to match the corresponding frequencies $\left\{\Omega_{\text {mod }}+\omega\right\}$ with the observed frequencies $\left\{\Omega_{\odot}\right\}$. Expression for $\omega=\omega(q)$ are derived by using perturbation theory, where the starting model is used as a zero-th order approximation. The corrections factor $q$ are then computed, assuming some regularity properties, so that the problem is mathematically well defined and/or unphysical solutions are avoided. 
c) The "helioseismological value" $Q_{\odot}$ is thus determined by adding the starting value and the correction:

$$
Q_{\odot}=Q_{\text {mod }}+q
$$

There are three independent sources of uncertainties in this process:

i)Errors on the measured frequencies, which - for a given inversion procedure - propagate on the value of $Q_{\odot}$.

ii)Residual dependence on the starting model: the resulting $Q_{\odot}$ is slightly different if one starts with different solar models. This introduces an additional uncertainty, which can be evaluated by comparing the results of several calculations.

iii)Uncertainty in the regularization procedure. Essentially this is a problem of extrapolation/parametrization. Different methods, equally acceptable in principle, yield (slightly) different values of $Q_{\odot}$.

It has to be remarked that, in view of the extreme precision of the measured frequencies, $\Delta \Omega_{\odot} / \Omega_{\odot} \lesssim 10^{-4}$ [14 [17], uncertainties corresponding to ii) and iii) are extremely important. We consider all these uncertainties, as well as the possible ways of combining them to estimate the global error.

All this will provide a framework for a quantitative comparison between solar models and helioseismology. In the final part of the paper we test the predictions of a few recent (standard) solar model calculations. We feel that similar test should be performed, when alternative solar models are proposed.

The paper is organized as follows:

A short review of the inversion methods is given in section 11 , where we also define the free parameters and determine their allowed ranges. The individual uncertainties mentioned above are operationally defined in section III, where possible definitions of the global errors are also discussed. In section IV the properties of the outer sun (photospheric helium abundance and quantities characterizing the bottom of the convective zone) are examined, and results are compared with previous analyses. Section $\nabla$ is devoted to the intermediate region $(x=0.2-0.65)$ whereas section $\mathrm{VI}$ concerns the energy production region. In section VII the results of a few recent solar models are compared with the available helioseismological constraints/tests.

The concluding section describes the main results of the paper, summarized in Figs. 2 and 3 and in Table 11 .

\section{HELIOSEISMIC DATA AND INVERSION METHODS: A SUMMARY}

\section{a) Observational data}

The helioseismic data that have been used for probing the solar interior structure are frequencies of acoustic oscillations (p-modes) corresponding to $Y_{l}^{0}$ spherical harmonics, with the degrees $l$ ranging from 0 to some 150. At low degrees there are typically 15 modes corresponding to rather high radial degrees, $n>10$. The total number of modes which has been used in structural inversions exceeds two thousands, which is only a small fraction of the total number of modes with measured frequencies. The latter number is higher by 
two orders of magnitude. However, modes corresponding to $l>150$ are not really useful for probing the structure of the sun's interior, because they propagate only in the shallow outermost layers and also because the accuracy of the corresponding frequencies is much worse. Tesseral $(m \neq 0)$ mode frequencies yield an information about the sun's internal rotation and magnetic field. In the present application, the only relevance of these data is to justify ignoring effects of the centrifugal and Lorentz forces on the radial structure.

The most exploited helioseismic data set is one from measurements at the Big Bear Solar Observatory in 1986 (BBSO86 [14]). This set contains no data for modes with $l<3$ and has been supplemented with data on $l=0-3$ modes from various sources. The most extensive data on such modes is from the network of automatic telescopes operated by the group of Birmingham University (BISON [15]). Recently, data covering the whole range of $l$-values became available from the LOWL instrument [16] and from the GONG network [17].

\section{b) Expression for $\omega=\omega(q)$}

The basic assumptions made in seismic probings of the sun's interior structure include hydrostatic equilibrium and adiabaticity of oscillations. In early inversions an asymptotic approximation for the radial eigenfunctions was used leading to a simple integral equation connecting the adiabatic sound speed, $c(R)$, to oscillation frequencies $\Omega_{l, n}$. However, this approximation is inadequate, especially for probing the deep interior and in most of the subsequent works numerical solutions have been adopted. The price is a need to use a SSM as a starting model about which the hydrostatic equations are linearized. There is an implicit assumption that the model provides a sufficently close description of the sun's structure. With this assumption, the variational principle following from the equation for the adiabatic oscillations, leads to an integral equation connecting the differences between the solar and the model functions describing radial structure (e.g. Ref. [18]) to the corresponding differences in mode frequencies. The equation, however, cannot be directly applied to infer the difference in the structural functions because the assumption of hydrostatic equilibrium of the mean structure as well as that of adiabaticity of oscillations break down near the surface. To account for these departures an ad hoc term of the form $F(\Omega) / I$, where $\Omega$ is the frequency an $I$ is a suitable moment of inertia, must be added . Such a form follows from the fact that there the radial eigenfunctions are nearly $l$-indepedent. The resulting formula may be written in the following form,

$$
\left(\frac{\Omega_{\odot}-\Omega_{\text {mod }}}{\Omega_{\text {mod }}}\right)_{j}=\int \mathcal{K}_{Q, j} \frac{q}{Q_{\text {mod }}} d x+\int \mathcal{K}_{\Gamma, j} \frac{\gamma}{\Gamma_{\text {mod }}} d x+\frac{F\left(\Omega_{\text {mod } j}\right)}{I_{j}}
$$

where $j \equiv(l, n)$ identifies the mode, $Q(x)$ is a structural function, $\Gamma=(d \log \mathrm{P} / d \log \rho)_{a d}$ is the adiabatic exponent, $x=R / R_{\odot}$ and:

$$
\begin{aligned}
& q=Q_{\odot}-Q_{\text {mod }} \\
& \gamma=\Gamma_{\odot}-\Gamma_{m o d}
\end{aligned}
$$

There is freedom in choosing $Q$. It could be density $\rho$, pressure $P$, or any combination of these quantities and their derivatives. All such functions are connected through the linearized mechanical equilibrium condition. 
Even with a very large number of Eqs. (2), equal to the number of all modes in the data set, a determination of the three functions: $q(x), \gamma(x)$ and $F(\Omega)$ is not possible without the additional assumption that the unknown functions vary slowly. One may eliminate one of the functions by making use of a thermodynamical relation $\Gamma=\Gamma(P, \rho, Y)$, where $Y$ is the fractional He abundance, and of two, well justified simplifications, which are:

(1) $\mathrm{Y}=\mathrm{Y}_{p h}$ in the convective envelope, and

(2) $\gamma=0$ in the radiative interior.

With these additional constraints the unknown function $\gamma$ is related with the unknown number $\mathrm{Y}_{p h}^{\odot}$. This approach was first adopted in [19] with the choice that the single directly determined function was $U=P / \rho$.

Thus Eqs. (2) are written in terms of the unknown function

$$
u(x)=U_{\odot}-U_{m o d},
$$

the function $F(\Omega)$ and of the unknown number $\mathrm{y}_{p h}=\mathrm{Y}_{p h}^{\odot}-\mathrm{Y}_{p h}^{\text {mod }}$. One advantage of this formulation of the inverse problem is that one obtains directly the surface abundance of He. We follow this approach here. Inversion without reference to thermodynamics has been done by Antia and Basu [20].

c) The regularized least square method and its free parameters

One way of making use of the assumption about the slow variability of $u / U_{m o d}$ and $F(\Omega)$ is a discretization in terms of known functions. Following [19], we use cubic splines in the first case and Legendre polynomials in the second case. The coefficients in these two representations together with $\mathrm{y}_{p h}$ are determined by the least-squares method. A simpleminded application of this method results in a solution for $u / U_{\bmod }$ which exhibits artificial oscillations. A cure to this problem is regularization (see e.g. [21]) which consists in adding to the usual $\chi^{2}$ an additional term. Here we minimize

$$
\chi_{\text {reg }}^{2}=\sum_{j=1}^{J}\left(\frac{\Omega_{\odot}-\Omega_{\text {mod }}}{\Delta \Omega_{\odot}}\right)_{j}^{2}+\frac{\lambda}{J} \int\left(\frac{d^{2}}{d x^{2}} \frac{u}{U_{\text {mod }}}\right)^{2} d x
$$

where $\mathrm{J}$ is the total number of the frequency data, $\Delta \Omega_{\odot}$ are the errors on the measured frequencies and $\lambda$ is a control parameter. This form of regularization is referred to as the second derivative smoothing. Alternative forms of regularization and influence of the choice of $\lambda$ were investigated in [22].

The regularization parameter $\lambda$ was chosen to be the minimum value that still suppresses the oscillations. Numerical experiments were conducted in which attempt was made to reproduce known differences in $u(x)$ between two solar models from differences in p-mode frequencies. The set of modes was the same as in the observational data with weights determined by the observational errors. The conclusion from these experiments was that for $x>0.1$ it is possible to reproduce $u(x)$ very accurately and independently of the choice of the type of the regularization. The results are also insensitive to $\lambda$ in a wide range of values. However in the inner core $(x<0.1)$ a regularization needed to avoid the oscillatory behaviour always flattened the real steep increase in $u / U_{\text {mod }}$. It became clear that the method is not applicable for probing this part of the sun. The reason is that only a small fraction of modes 
in the data sets exibits any measurable sensitivity to the sound speed changes in the inner core and the effect of regularization dominates over the data.

Uncertainities on $\mathrm{y}_{p h}$ and $u(x)$, as well as on other indirectly determined structure functions, following from observational errors, are best estimated by random number simulation having normal distribution with the half-width given by $\Delta \Omega_{\odot}$. There is an uncertainty following from the freedom in: choice of the regularization parameter $\lambda$, number of bins, $n_{b}$, and number of the Legendre polynomials, $n_{F}$. This has been discussed in [19,22,23]. Changes in $n_{b}$ may always be compensated by changes in $\lambda$ and will not be discussed here. Admissible ranges for $\lambda$ and $n_{F}$ follow from considering the behavior of $\chi_{r e g}^{2}$. An increase in $\lambda$, as seen in Eq. (5), causes an increase in $\chi_{\text {reg }}^{2}$, the same effect being caused by the decrease of $n_{F}$. Specifying the maximum departure from the minimum value $\chi_{\text {reg }}^{2(M I N)}$ we can set an upper bound for $\lambda$ and a lower bound for $n_{F}$. Concerning $\lambda$, one adds the requirement of avoiding artificial oscillations. Dealing with the real data we may still distinguish the artificial oscillations from features implied by data. The former have the half-wavelength equal to the bin length and their amplitude increases sharply with the decrease of $\lambda$. Appearence of the oscilllation sets a lower bound for $\lambda$.

Let us summarize the role of the parameters of this method, which will be referred to as the regularized least square (RLS) method:

i) There are two parameters, $\lambda$ and $n_{F}$.

ii) The seismic model of the sun is obtained by taking $\lambda=0.001$, as this is the minimal value for which unphysical oscillations are avoided, and $n_{F}=20$ as this corresponds to the minimal degree of the polynomial such that $\chi_{\text {reg }}^{2}$ is stable.

iii)For this set of parameters, by using the "model S" of Ref. [24] (hearafter referred to as JCD), as a starting model, we obtain $\chi_{\text {reg }}^{2(M I N)}=2.5$, which provides an indication of the fit quality.

iv) For the parameters $\lambda$ and $n_{F}$ we consider as acceptable those values which yield a $\chi_{r e g}^{2}$ not substantially degraded with respect to the minimum. As a prescription borrowed from statistics we accept the parameters if $\chi_{r e g}^{2} \leq \chi_{\text {reg }}^{2(M I N)}+1$. Numerically we found that this implies $\lambda \leq 0.1$ and $n_{F} \geq 10$.

d) Methods and parameters for the solar core

As already remarked, the method described above cannot be used for probing the core within $x=0.1$. However, a fairly accurate seismic sounding of that region should be possible.

It was found [23] that frequencies for some 30 solar p-modes were changed by more than $\Delta \Omega_{\odot}$ when $U$ in this innermost part of the core was changed by just one percent. For that region the optimal localized averaging (OLA) method is a better choice. In this method we do not try to determine functions. Instead, we try to determine mean values weighted with (possibly narrow) kernels centered at selected $\left\{x_{0}\right\}$ values. We consider a linear superposition of individual kernels for the modes present in the data set,

$$
K_{u}\left(x_{0}, x\right)=\sum_{j}^{J} c_{j}\left(x_{0}\right) \mathcal{K}_{u, j}(x)
$$

and determine the coefficients $c_{j}$ at selected points. An application of the classical method 
of Backus \& Gilbert [25] to helioseismic data was described by Gough \& Thompson [18]. The subtractive optimal localized averaging (SOLA) method adopted in [22] and used also in this paper was developed by Pijpers \& Thompson [26]. Here one tries to construct kernels $K_{u}\left(x_{0}, x\right)$ which are as close as possible to Gaussians, $G$, centered in $x_{o}$ and characterized by their half-widths at half-maximum, $w$. Subject to minimization is the quantity

$$
\int\left[K_{u}-G\left(\frac{x-x_{0}}{w}\right)\right]^{2} d x+\mu \sum_{j}^{J} c_{j}^{2} \Delta \Omega_{\odot j}^{2} .
$$

The second term, with a trade-off parameter $\mu$, is added to avoid large error magnification. For a specified $w$, larger $\mu$ leads to smaller errors in the localized mean but the kernels may differ significantly from Gaussians and therefore $w$ cannot be regarded as the measure of localization. Once the coefficients $c_{j}$ are determined the localized averages are:

$$
<\frac{u}{U}>=\sum_{j} c_{j}\left(\frac{\Omega_{\odot}-\Omega_{\text {mod }}}{\Omega_{\text {mod }}}\right)_{j} \pm \sqrt{\sum_{j} c_{j}^{2} \Delta \Omega_{\odot j}^{2}}
$$

A comparison of results of inversions with the two methods, given in [22,23], shows a very good agreement everywhere except for the inner core. Results obtained with the SOLA method are more realistic because it does not introduces an artificial smoothing. Thus, for $x>0.1$ we may rely on the functional form of $u(x)$ as obtained by means of the RLS method. However, for a reliable and accurate probing of the inner core a different approach is required. In a hybrid method of seismic model construction developed in [22], the RLS method is used to determine $F(\Omega), \mathrm{y}_{p h}$ and $u / U_{\text {mod }}$ in the $\left[x_{f}, 1\right]$ interval, whereas the SOLA method is used to determine a single average value in the inner core around the point $x_{0}$. The effect of $F(\Omega)$ and $\mathrm{y}_{p h}$ is removed from the data. The function $u / U_{\bmod }$ in the $\left[0, x_{f}\right]$ range is then represented as a three term power series in $x^{2}$ thereby satisfying the boundary condition at $x=0$. The three coefficients in the series are determined using the average SOLA value with the true kernel around $x_{0}$ and the continuity conditions at $x=x_{f}$ for $u / U_{\text {mod }}$ and its derivative. The method introduces its own uncertainties resulting from some freedom in choosing $x_{0}$ and $x_{f}$. Regarding the role of the parameters of the hybrid method we point out that:

i)The constraint on $x_{f}$ is that the point should be located in the region where the SOLA values agree within the observational errors with the values inferred by the RLS method. This occurs for $x_{f i t} \geq 0.075$. We shall explore the effects of varying $x_{f}$ in the range [0.075 $-0.125]$.

ii)We shall also study the effect of varying $x_{0}$, while $x_{f}$ is kept at the central value $x_{f}=0.1$. The range for $x_{0}$ is suggested by two conditions: at too small values there is not enough information. On the other hand, when $x_{0}$ is too large and gets close to $x_{f}$ there is no arm for extrapolating the information to small distances. As a consequence, somehow empirically, we chose $x_{0}$ in the range $[0.04-0.06]$ and we adopt $x_{0}=0.05$ as the "best" value.

iii) The choice of the trade-off parameter, $\mu$, for the hybrid method should be such as to minimize errors in $u / U_{\text {mod }}$ implied by the measurement errors; we chose $\mu=0.001$. 


\section{PARTIAL UNCERTAINTIES AND GLOBAL ERRORS}

We shall estimate how quantities $Q$ characterizing the solar structure are changed when some input of the helioseismological determination are varied, one at a time. As a reference, we consider the values $Q_{\odot}$ obtained with the seismic model described in the previous section: frequencies are kept at the central measured values, the JCD model is used as the starting model, and the inversion parameters are kept at the "best" values $\lambda=0.001, n_{F}=20$, $x_{f i t}=0.1$ and $x_{0}=0.05$. (The results of the seismic model are presented in Table II], second column, and Table [V] again second column.)

1) Observational errors. We consider the effect of varying the frequencies from the central measured values, within their observational errors. We took random choices of frequencies within $\pm \Delta \Omega_{\odot}$ and evaluated the corresponding values of $Q$. In this way we found maximal (minimal) acceptable values, $Q_{\text {sup }}\left(Q_{\text {inf }}\right)$. We define the allowed range as:

$$
(\delta Q)_{\text {exp }}=Q_{\text {sup }}-Q_{\text {inf }}
$$

2)Starting model. For the central values of the observed frequencies and the best values of the inversion parameters, we study the differences resulting from using different starting models. We remind that JCD includes diffusion of helium and heavier elements, uses the Livermore equation of state and the Livermore opacity tables. To assess the relevance of these inputs, we performed inversion by using:

a) a model (SUN24l) with the same equation of state and opacities, but without diffusion (modified version of "model 0" from Ref. 23] ).

b) a model (SUN24) with the same opacities, the MHD equation of state and without diffusion ("model 0" from Ref. 23] ).

Exactly as before, for each quantity $Q$ we evaluated the allowed range, $(\delta Q)_{\text {mod }}$.

3)Inversion method. As explained in the previous section, two different inversion methods are adopted, depending on the value of the radial coordinate. For $x \geq 0.1$, we use the RLS method and consider the effect of varying $\lambda$ and $n_{F}$ separately. The parameter $\lambda$ is varied in the range $0.001-0.1$, see section $\mathbb{1 1}$. The resulting variation (maximum minus minimum in the explored range) induced on the quantity $Q$ will be denoted as $(\delta Q)_{\lambda}$. The other parameter $n_{F}$ is varied in the range 10-20, see again section [1], and the resulting variation is denoted as $(\delta Q)_{n_{F}}$.

For the inner region we use the hybrid method: the function $U(x)$ is determined by the requirement that an interpolating polynomial matches the results of the SOLA method around $x_{0}$ with that of the RLS method at $x_{f}$. As discussed in section 【, we consider $x_{f}$ in the range $[0.075-0.125]$ and $x_{0}$ in the range $[0.04-0.06]$.

Although we are confident that our analysis is rather exhaustive, there is no well defined rule as to translate the differences $(\delta Q)_{k}$ into a global error $\Delta Q$. Let us present two, somehow extreme, attitudes:

a)The statistical approach. If one believes that, for each parameter, all possible values lie in the interval $Q_{k}^{\text {inf }}-Q_{k}^{\text {sup }}$, one can interpret $\pm 1 / 2(\delta Q)_{k}$, as a partial error. Furthermore, one can assume that the partial uncertainties somehow compensate each other, and use 
the combination rule for the statistical fluctuations of independent variables, which (for Poisson-Gauss distribution) yields:

$$
(\Delta Q)_{s t a}= \pm \frac{1}{2} \sqrt{\sum_{k}(\delta Q)_{k}^{2}}
$$

b) The conservative approach. May be that the parameter variation was not exhaustive, and what we found as extrema are not really so, but actually are quite acceptable values. In view of this, let us double the interval we found and interpret $\pm(\delta Q)_{k}$, as partial errors. Furthermore, let us be really conservative assuming that errors add up linearly. In conclusion, this gives:

$$
\left(\Delta Q_{\text {con }}\right)= \pm \sum_{k}\left|(\delta Q)_{k}\right|
$$

In the following sections, we shall present both error estimates. The first one is reasonable when most of the error corresponds to the statistical uncertainty of the measured frequencies. Actually in our case the other errors (from the residual model dependence and/or inversion method) are generally dominant. Clearly they are of a more subtle nature, a sort of systematic errors. For this reason, we feel more confident with the conservative error estimate, Eq. (11).

Before concluding this section, let us briefly consider the question of the error correlation, which is important when considering the helioseismological implications on two quantities $Q^{(1)}$ and $Q^{(2)}$ (e.g. $\mathrm{Y}_{p h}$ and $R_{b}$ ). If the respective errors are $\Delta Q^{(1)}$ and $\Delta Q^{(2)}$, we shall consider a solar model as acceptable if the predictions lie within the rectangle $Q^{(1)} \pm \Delta Q^{(1)}$, $Q^{(2)} \pm \Delta Q^{(2)}$, i.e we shall treat errors as uncorrelated, whereas actually there is a correlation: as an example, the extremum $Q^{(1)}+\Delta Q^{(1)}$ most likely corresponds to a set of parameters which does not allow - say $-Q^{(2)}-\Delta Q^{(2)}$. The helioseismologically allowed area should be an ellipsis contained inside the rectangle. By choosing the rectangle as the allowed area, one is thus overestimating the error, which again corresponds to a conservative attitude.

\section{PROPERTIES OF THE CONVECTIVE ENVELOPE}

\section{A. The photospheric Helium abundance}

Knowledge of the helium abundance in the sun is of fundamental importance both to cosmology and to solar structure theory. Unfortunately, it is impossible to measure it, even in the atmosphere, by means of standard spectroscopy. A helioseismological method of determining $Y_{p h}$ was first suggested by Gough [27] and developed in Refs. [28] and [29]. The value of $\mathrm{Y}_{p h}$ inferred for the sun, however, was not published, because it was feared that it might be too sensitive to systematic errors in the data that were available at the time. In [12] an alternative method was developed. Unlike the former one, it does not rely on the validity of p-mode asymptotics. Instead, it requires the validity of linearization about a reference model.

Several helioseismological determinations of $\mathrm{Y}_{p h}$ have been published since 1991. The various estimates, which are collected in Table $\llbracket$, often differ among each other by more than 
the quoted errors, see Fig. 1. Actually, these latter often reflect only the uncertainties of the measured frequencies, whereas the extracted value of $\mathrm{Y}_{p h}$ depends on the inversion method and on the physical inputs, see the discussion in Refs. [30] and [31]. Most of the estimates

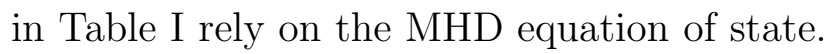

By omitting the highest value by Dappen et al. [32] (the same authors later published significantly smaller values [31]) the total range of helioseismological determinations is:

$$
\mathrm{Y}_{p h}=0.226-0.260
$$

Our reference estimate is $\mathrm{Y}_{p h}^{\odot}=0.249$ (we remark the we rely on the Livermore equation of state, which should be more accurate than MHD). Concerning the allowed range, the result of our conservative analysis, shown in Table [1], implies:

$$
\mathrm{Y}_{p h}=0.238-0.259
$$

which substantially overlaps with the previous range. From the same table one sees that experimental errors are of minor relevance, the value of $\mathrm{Y}_{p h}$ being manly sensitive to the

choice of the starting model; as discussed in Refs. [30] and [31], the effect of the equation of state is dominant.

\section{B. The bottom of the convective zone}

As emphasized in Ref. [13], the transition of the temperature gradient between being subadiabatic and adiabatic at the base of the solar convective zone gives rise to a clear signature in the sound speed. Helioseismic measurements of the sound speed therefore permit a determination of the location of the base of the convective zone.

In Ref. [13] the following ranges were derived:

$$
\begin{gathered}
R_{b} / R_{\odot}=0.710-0.716 \\
c_{b}=(0.221-0.225) \mathrm{Mm} / \mathrm{s},
\end{gathered}
$$

where the quoted intervals include the uncertainty resulting from the inversion technique. The estimate of $R_{b}$ has been confirmed in Refs. [33, 30].

Actually, within the present uncertainty $R_{b}$ and $c_{b}$ are not independent [13]. As well known, the lower part of the convective zone is very close to being adiabatically stratified, the adiabatic exponent $\Gamma$ being close to $5 / 3$, hence

$$
P \propto \rho^{5 / 3}
$$

Furthermore, throughout the same region, in the hydrostatic equation one can approximate $M_{x}$ (the mass within $x$ ) with the total mass:

$$
\frac{d P}{d x}=-\frac{G M_{\odot}}{R_{\odot} x^{2}} \rho
$$


In terms of $U=P / \rho$ the two equations give:

$$
\frac{d U}{d x}=-\frac{2}{5} \frac{G M_{\odot}}{R_{\odot} x^{2}} \rho
$$

and consequently

$$
U(x) \simeq \frac{2}{5} \frac{G M_{\odot}}{R_{\odot}}(x-1)
$$

which shows that $U$ (hence $\mathrm{c}=\sqrt{5 / 3 U}$ ) is uniquely known as a function of $\mathrm{x}$. In view of the approximations which have been used $\left[M_{x} \simeq \mathrm{M}_{\odot}, \Gamma \simeq 5 / 3\right]$, Eq. (19) is accurate to the percent level, and this is adequate in comparison with the error of the helioseismological determination of $c_{b}$, see Eq. (15).

On the other hand, the density $\rho_{b}$ at the bottom of the convective zone is an independent quantity. Actually, from the above equations $\rho(x)$ in the convective zone is determined up to a scaling factor. The helioseismological determination of $\rho_{b}$ fixes such a factor. Thus $\rho_{b}$ is also an interesting quantity, which however received little attention in the past.

We derived $\rho_{b}$ from $U(x)$ by integrating the hydrostatic equilibrium equation:

$$
\frac{1}{\rho} \frac{d \rho}{d x}=-\frac{1}{U}\left[\frac{d U}{d x}+\frac{G M_{x}}{R_{\odot} x^{2}}\right],
$$

together with

$$
\frac{d M_{x}}{d x}=4 \pi \rho R_{\odot} x^{2}
$$

Thus $\rho_{b}$ is sensitive to all the uncertainties of the inversion method, including those of small $x$ region.

Our analysis, see again Table [1], yields:

$$
R_{b} / R_{\odot}=0.708-0.714
$$

essentially confirming the result of [13]. Note that again the main sensitivity is to the starting model. Concerning the density, we find:

$$
\rho_{b}=(0.185-0.199) \mathrm{g} / \mathrm{cm}^{3}
$$

Also in this case, a large fraction of the error comes from the starting model.

\section{THE INTERMEDIATE REGION}

The essential output of helioseismology is the reconstruction of the sound speed profile. Our discussion is in terms of the related quantity $U$; as well known, $c^{2}=\Gamma U$ and below the convective zone $\Gamma=5 / 3$ with an accuracy of $10^{-3}$ or better.

By using the RLS method, it is possible to derive directly the profile of $U$ as a function of the radial coordinate throughout all the sun, except for the inner region $(x<0.1)$. The 
results (values of $U$, partial uncertaintes and global errors), are shown in Table $\amalg \mathrm{V}$ and Fig. 2 and are summarized in Table [I].

It is convenient to consider an intermediate solar region: $0.2<x<0.65$. The upper limit is established by requiring that it is well below the transition to the convective zone, which we discussed above. The lower limit is chosen so as to exclude the region of energy production (see next section). For this region the following comments are relevant:

a)Each of the individual uncertainties nowhere exceeds $2 \%$.

b) Uncertainties from the accuracy on the measured frequencies are of minor relevance with respect to the residual model dependence and to the sensitivity to the inversion parameters.

c)All in all, even with the most conservative estimates, the helioseismological determination is extremely accurate: $|\Delta U / U| \leq 5^{\circ}$ \%o throughout the explored region.

\section{THE ENERGY PRODUCTION REGION}

As well known, most of the energy and of solar neutrinos originate from the innermost part of the sun. According to SSM calculations, see e.g. Refs. [3, 34, about $94 \%$ of the solar luminosity and $93 \%$ of the pp neutrinos are produced within $x<0.2$, the region which we analyse in this section.

Our results, summarized in Fig. 2 and Table [1, deserve the following comments:

i) In the region $0.1<x<0.2$, where the RLS method holds, the error intrinsic to the inversion method is dominant, and the global uncertainty, which worsens as one is going deeper into the sun, reaches $\pm 1 \%$.

ii) At even smaller radii, where the hybrid method is used to determine the function $U(x)$, a large fraction of the error comes from the choice of $x_{f}$ and of $x_{0}$, whereas the residual dependence on $\lambda$ and $n_{F}$ is rather weak. The result is also essentially unsensitive to the starting model. On the other hand, errors on frequencies become important. This obviously corresponds to the fact that p-modes do not penetrate in the solar core, and consequently the information one can extract from available experimental results is limited.

iii) We remind that the production of ${ }^{7} \mathrm{Be}$ neutrinos is limited to a small region close to the solar center, the maximal production occurring at:

$$
x_{B e}=0.06
$$

according to [3]. The ${ }^{8} \mathrm{~B}$ neutrinos are generated even closer to the center, the maximal production being at [3]:

$$
x_{B}=0.04
$$

(The exact locations of these maxima are somehow model dependent: for instance, in the solar model with helium and metal diffusion of Ref. [34, hearafter referred to as BP95, one has $x_{B e} \simeq 0.055$ and $\left.x_{B} \simeq 0.045\right)$. One sees from Table II that $U\left(x_{B e}\right)$ and $U\left(x_{B}\right)$, as derived from the hybrid method, are globally known with an accuracy of about $2 \%$. The average values around $x_{B e}$ and $\left(x_{B}\right)$ given by the SOLA method are in agreement, within the quoted errors, with the results of the hybrid method. 


\section{HELIOSEISMOLOGY AND STANDARD SOLAR MODELS}

A few recent solar models will now be compared with the helioseismological information derived in the previous sections.

In this respect, the predicted vs. observed properties of the convective envelope are particularly interesting, see Table III and Fig. 3. All solar models neglecting diffusion cannot account for $\mathrm{Y}_{p h}$ and for the properties of the bottom of the convective zone $\left(R_{b}\right.$ and $\left.\rho_{b}\right)$. Models with diffusion of helium and heavier elements are much closer to the helioseismological determinations. Among these, BP95 and JCD yield values of $\mathrm{Y}_{p h}, \mathrm{R}_{b}$ and $\rho_{b}$ in good agreement with helioseismological predictions.

For understanding what is going on, let us concentrate on our own solar calculations. FRANEC96 [35] is our most recent solar model, including diffusion of $\mathrm{He}$ and heavier elements and using the Livermore EOS and the new Livermore opacities for 19 elements [36] according to the composition of [37]. If diffusion is switched off, the resulting model (FRANEC96-ND) is grossly inconsistent with helioseismology. FRANEC96 looks much better in this respect. Hovewer it slightly underestimates the depth of the convective zone; as this is too shallow, the density at the bottom is too small (and correspondingly the sound speed is somehow underestimated). If we use the older Livermore opacities calculated for 12 elements, the resulting model (FRANEC96-OLD) is in good agreement with helioseismology.

In Fig. Twe show the difference between $U$ as predicted by selected solar models and the helioseismological determination, normalized to this latter. We remark that BP95 and JCD model are in agreement with helioseismology everywhere.

SUN24, the model without diffusion of Ref. [23] yields a good profile of $U(x)$. We remind, hovewer, that it fails in predicting the properties of the convective envelope. This shows that the two approaches (profile of $U$ and properties of the convective envelope) are complementary and both important.

FRANEC96 underestimates $U$ by about $1 \%$ near the transition between the convective and radiative region, as already discussed. On the other hand, FRANEC96-OLD, as well as other models using older versions of the Livermore opacities (BP95, JCD and SUN24) look better. This does not imply some deficiency of the latest opacities. The important point, however, is that helioseismology can discriminate between models based on opacities which differ just by a few percent. This is best seen in Fig.5 where the profile of $U$ according to FRANEC96 and FRANEC96-OLD are compared, together with the relative differences $\Delta k / k$ between opacities, these latter being calculated at any point $x$ for the same value of $\rho$, $\mathrm{T}$ and chemical composition (given by FRANEC96-OLD).

In conclusion, it seems to us that all these models with diffusion essentially pass the

helioseimological tests. Similar conclusions have been recently reached in Ref. [48], where the inconsistency of mixed solar models with helioseismology is also discussed.

\section{CONCLUDING REMARKS}

We summarize here the main points of this paper:

- An extensive an possibly exhaustive investigation of uncertainties in helioseismic determinations of solar properties has been performed. 
- The accuracy on quantities characterizing the convective envelope $\left(\mathrm{Y}_{p h}, R_{b}\right.$ and $\left.\rho_{b}\right)$ has been studied, see Table II, as well as that on the squared isothermal sound speed, $U=P / \rho$, along the solar profile, see Fig. 2 .

- Only recent standard solar models including diffusion of He and heavier elements predict properties of the convective envelope in agreement with helioseismology, see Table III and Fig. 3 .

- The same models also predict for $U(x)$ values consistent with the helioseismic determination all over the solar profile, see Fig. 4 .

Solar models built ad hoc so as to solve the solar neutrino problem should be at least as successful.

\section{ACKNOWLEDGMENTS}

W.A.D. is grateful to the director and the staff of the Osservatorio Astronomico di Collurania (Teramo) for their hospitality and he gratefully acknowledges the financial support from Laboratori Nazionali del Gran Sasso of INFN. We appreciate useful discussion with V. Berezinsky and V. Castellani. We are grateful to F. Ciacio for his help in developing the version of FRANEC including diffusion of helium and heavier elements. We thank S. Basu, J. Christensen-Dalsgaard, A. Dar, R. Sienkiewicz and S. Turck-Chièze for providing us with detailed results of their solar models. We thank A.A. Pamyatnykh and P.R. Goode who collaborated with W.A.D. on development of the inversion codes used in present work. This work was partially supported by Ministero dell' Università e della Ricerca Scientifica. 


\section{REFERENCES}

[1] J. N. Bahcall, "Neutrino Astrophysics", Cambridge University Press, Cambridge, 1989.

[2] V. Berezinsky, Comm. Nucl. Part. Phys. 21 (1994) 249.

[3] V. Castellani, S. Degl'Innocenti, G. Fiorentini, M. Lissia and B. Ricci, Phys. Rep. (1996) to appear.

[4] R. Davis Jr., D. S. Harmer and K. C. Hoffman, Phys. Rev. Lett. 20 (1968) 1205;

B. T. Cleveland et al., Nucl. Phys. B (Proc. Suppl.) 38 (1995) 47, and Refs. therein.

[5] K. S. Hirata et al., Phys. Rev. Lett. 63 (1989) 16;

Y. Fukuda et al., Phys. Rev. Lett. 77 (1996) 1683 and Refs. therein.

[6] A. I. Abazov et al., Phys. Rev. Lett. 67 (1991) 3332;

SAGE Collaboration, J. N. Abdurashitiv et al., Nucl. Phys. B (Proc. Suppl.) 48 (1996) 299, and Refs. therein.

[7] GALLEX Collaboration, P. Anselmann et al., Phys. Lett. B 327 (1994) 377;

GALLEX Collaboration, P. Anselmann et al., Phys. Lett. B 357 (1995) 237, and Refs. therein.

[8] D. O. Gough, J. W. Leibacher, P. H. Scherrer and J. Toore, Science 272 (1996) 1281.

[9] W. Unno, Y. Osaki, H. Ando, H. Saio and H. Shibahashi, "Non radial oscillations of stars", University of Tokio Press, Tokio 1989.

[10] D. 0. Gough and J. Toomre, Ann.Rev.Astron.Astroph. 29 (1991) 627.

[11] S. V. Vorontsov, V. A. Baturin and A. A. Pamyatnykh, Nature 349 (1991) 49.

[12] W. A. Dziembowski, A. A. Pamyatnykh and R. Sienkiewicz, Mon. Not. R. Astron. Soc. 249 (1991) 602.

[13] J. Christensen-Dalsgaard, D. O. Gough and M. J. Thompson Ap. J. 378 (1991) 413.

[14] K. G. Libbrecht, M. F. Woodard and J.M. Kaufman, Ap. J. Suppl. 74 (1990) 1129.

F. P. Pijpers and M. J. Thompson, Astron. Astr. 262 (1992) L33.

[15] Y. Elsworth, R. Howe, G. R. Isaak and C. P. McLeod, R. New, Ap. J. 434, (1994) 801.

[16] S. Tomczyk, K. Streander, G. Card, D. Elmore, H. Hull and A. Cacciani, Sol. Phys. 159 (1995) 1.

[17] J. E. Harvey et al, Science, 272 (1996) 1284.

[18] D. O. Gough and M. J. Thompson, 1991, in "The Interior and Atmosphere of the Sun", A. N. Cox and M. S. Matthews eds., University of Arizona Press, p. 501.

[19] W. A. Dziembowski, A. A. Pamyatnykh and R. Sienkiewicz, Mon. Not. R. Astron. Soc. 244 (1990) 542.

[20] H. M. Antia and S. Basu, Astron. Astr. Suppl. 107 (1994) 421.

[21] I. J. D. Craig and J. C. Brown, 1986, "Inverse Problems in Astronomy: A Guide to Inversion Strategies for Remote Sensed Data", Bristol: A. Hilger.

[22] W. A. Dziembowski, P. R Goode, A. A. Pamyatnykh and R. Sienkiewicz Ap. J. 432 (1994) 417.

[23] W. A. Dziembowski, Bull. Astr. Soc. India 24 (1996) 133.

[24] J. Christensen-Dalsgaard et al. , Science 272 (1996) 1286.

[25] G. Backus and F. Gilbert, Geophys. J. Roy. Astron. Soc. 16 (1968) 169.

[26] F. P. Pijpers and M. J. Thompson, Astron. Astr. 262 (1992) L33.

[27] D. O. Gough, Mem. Soc. Astr. Italiana 55 (1984) 13. 
[28] W. Dappen and D.O. Gough, in "Theoretical problems in stellar stability and oscillations" p. 264, M. Gabriel and A. Noels eds., Institute d'Astrophysique, Liege 1984.

[29] W. Dappen, D.O. Gough and M. J. Thompson, in "Seismology of the sun and sun-like stars, p. 505 , E.J. Rolfe ed., ESTEC, Noordwijk 1988.

[30] O. Richard, S. Vauclair, C. Charbonnel and W. A. Dziembowski, preprint e-archive astro-ph/9604009, Astron. Astrophys. (1996), to appear.

[31] A. G. Kosovichev, J. Christensen-Dalsgaard, W. Dappen, W. A. Dziembowski, D. O. Gough and M. J. Thompson, Mon. Not. R. Astron. Soc. 259 (1992) 536.

[32] W. Dappen, D. O. Gough, A. G. Kosovichev and M. J. Thompson, in "Challenges to the theories of the structure of moderate-mass stars", D. O. Gough and J. Toomre eds., Springer, Heidelberg 1991.

[33] J. A. Guzik and A. N. Cox, Ap. J. 411 (1993) 394.

[34] J. N. Bahcall and M. H. Pinsonneault, Rev. Mod. Phys 67 (1995) 781.

[35] F. Ciacio, S. Degl'Innocenti and B. Ricci, Astron. Astrophys. Suppl. (1996) to appear.

[36] C.A. Iglesias and F.J. Rogers, Ap. J. 464 (1996) 943

[37] N. Grevesse and A. Noels in "Origin and Evolution of the elements", N. Prantzos, E. Vangioni Flam and M. Casseèds., Cambridge University Press, p.15; N. Grevesse and A. Noels, Phys. Scripta T47 (1993) 133.

[38] W. A. Dziembowski, P. R. Goode, A. A. Pamyatnykh and R. Sienkiewicz, Ap. J. 432 (1994) 417.

[39] F. P. Hernandez and J. Christensen-Dalsgaard, Mon. Not. R. Astron. Soc. 269 (1994) 475.

[40] H. M. Antia and S. Basu, Ap. J. 426 (1994) 801.

[41] D. O. Gough et al., Science 272 (1996) 1296.

[42] A. N. Cox, J. A. Guzik and R. B. Kidman, Ap. J. 342 (1989) 1187.

[43] C. R. Proffitt, Ap. J. 425 (1994) 849.

[44] A. Dar and G. Shaviv, Ap. J. 468 (1996) 933.

[45] S. Basu, J. Christensen-Dalsgaard, J. Schou, M. J. Thompson and S. Tomczyk, Ap. J. (1996) to appear.

[46] S. Turck-Chièze and I. Lopes, Ap. J. 408 (1993) 347.

[47] J. N. Bahcall and M. H. Pinsonneault, Rev. Mod. Phys. 64 (1992) 885.

[48] J. N. Bahcall, M. H. Pinsonneault, S. Basu and J. Christensen-Dalsgaard, 1996, submitted to Phys. Rev. Lett. 


\section{TABLES}

TABLE I. Helioseismological determinations of the photospheric He abundance, $\mathrm{Y}_{p h}$.

\begin{tabular}{lc}
\hline \hline Ref. & $Y_{p h}$ \\
\hline Dappen (1991) [32] & $0.268 \pm 0.010$ \\
Vorontsov (1991) [11] & $0.250 \pm 0.010$ \\
Dziembowski (1991) [12] & $0.234 \pm 0.005$ \\
Kosovichev (1992) [31] & $0.232 \pm 0.006$ \\
Cox (1993) 33] & $0.240 \pm 0.005$ \\
Dziembowski (1994) [38] & $0.24295 \pm 0.0005$ \\
Hernandez (1994) 39] & $0.242 \pm 0.003$ \\
Antia (1994) 410] & $0.252 \pm 0.003$ \\
Gough (1996) [41] & $0.248 \pm 0.005$ \\
RVCD96 [30] & $0.250 \pm 0.005$ \\
\hline \hline
\end{tabular}

TABLE II. For the indicated quantities $Q$ we present the helioseismological reference values $Q_{\odot}$, the relative partial uncertainties $(\delta Q / Q)_{k}$ corresponding to experimental errors $($ exp $)$, model dependence $(\mathrm{mod})$, and to the parameters used in the inversion method $\left(\lambda, n_{F}, x_{f i t}\right.$ and $\left.x_{o}\right)$. Global errors $\Delta Q / Q$, estimated according to the statistical (sta) and the conservative (con) approach, see Eqs. (10) and (11), are also shown. All uncertainties and errors are in $\%$. In the fifth and sixth row, for the quantity $U=P / \rho$ the values of the partial uncertainties and of the global errors are the maxima in the indicated interval. In the last two rows the results on $U$ at points representative of the ${ }^{7} \mathrm{Be}$ and ${ }^{8} \mathrm{~B}$ neutrino production, Eqs. (24) and (25), have been derived by using the hybrid method.

\begin{tabular}{|c|c|c|c|c|c|c|c|c|c|}
\hline$Q$ & $Q_{\odot}$ & $\left(\frac{\delta Q}{Q}\right)_{e x p}$ & $\left(\frac{\delta Q}{Q}\right)_{\bmod }$ & $\left(\frac{\delta Q}{Q}\right)_{\lambda}$ & $\left(\frac{\delta Q}{Q}\right)_{n_{F}}$ & $\left(\frac{\delta Q}{Q}\right)_{x_{f i t}}$ & $\left(\frac{\delta Q}{Q}\right)_{x_{o}}$ & $\left(\frac{\Delta Q}{Q}\right)_{s t a} \mid$ & $\left(\frac{\Delta Q}{Q}\right)_{c o n}$ \\
\hline$\overline{Y_{p h}}$ & 0.249 & 2.4 & 27 & 6 & 7 & & & 14 & 42 \\
\hline$R_{b} / R_{\odot}$ & 0.711 & 0.1 & 4 & 0.03 & 0.01 & & & 2 & 4 \\
\hline$\rho_{b}\left[\mathrm{~g} / \mathrm{cm}^{3}\right]$ & 0.192 & 3 & 16 & 4 & 6.4 & 2.5 & 4.8 & 9.4 & 37 \\
\hline$U(0.2<x<0.65)$ & & $<1$ & 2 & 1.3 & 1.4 & & & 1.4 & 5 \\
\hline$U(0.1<x<0.2)$ & & $<1$ & 1.6 & 2.5 & 3.0 & 1.5 & & 2.3 & 9.4 \\
\hline$\overline{U\left(x_{B e}\right)\left[10^{15} \mathrm{~cm}^{2} \mathrm{~s}^{-2}\right]}$ & 1.56 & 2.7 & 1.5 & 1.7 & 2.1 & 3.5 & 6.6 & 4.3 & 18 \\
\hline$U\left(x_{B}\right)\left[10^{15} \mathrm{~cm}^{2} \mathrm{~s}^{-2}\right]$ & 1.55 & 4.8 & 1.3 & $<1$ & 1.5 & 3.7 & 11 & 6.6 & 24 \\
\hline
\end{tabular}


TABLE III. Helioseismological determinations and solar model predictions for the properties of the convective envelope

\begin{tabular}{|c|c|c|c|c|}
\hline model & Ref. & $R_{b} / R_{\odot}$ & $\mathrm{Y}_{p h}$ & $\rho_{b}\left[\mathrm{~g} / \mathrm{cm}^{3}\right]$ \\
\hline \multicolumn{5}{|c|}{ Models without diffusion: } \\
\hline FRANEC96-ND & 35 & 0.728 & 0.261 & 0.156 \\
\hline BP95-ND & 34] & 0.726 & 0.268 & 0.157 \\
\hline CGK89-ND & 42 & 0.714 & 0.291 & \\
\hline P94-ND & 43 & 0.726 & 0.270 & \\
\hline RVCD96-ND & 30 & 0.725 & 0.278 & 0.166 \\
\hline DS96-ND & 444] & 0.731 & 0.285 & \\
\hline BCDSTT-ND & 45 & 0.721 & 0.276 & $0.171^{*}$ \\
\hline TCL & 46 & 0.725 & 0.271 & $0.166^{*}$ \\
\hline SUN24 & 23 & $0.716^{*}$ & $0.283^{*}$ & $0.184 *$ \\
\hline SUN241 & & $0.714^{*}$ & $0.282 *$ & 0.186 * \\
\hline \multicolumn{5}{|c|}{ Models with He diffusion: } \\
\hline BP92 & 47 & 0.707 & 0.247 & 0.197 \\
\hline P94 & 43 & 0.710 & 0.246 & \\
\hline BCDSTT & 45 & 0.707 & 0.246 & $0.199^{*}$ \\
\hline \multicolumn{5}{|c|}{ Models with He and $\mathrm{Z}$ diffusion: } \\
\hline FRANEC96 & 35 & 0.716 & 0.238 & 0.181 \\
\hline FRANEC96-OLD & & 0.713 & 0.242 & 0.187 \\
\hline BP95 & 34 & 0.712 & 0.247 & 0.187 \\
\hline CGK89 & 42 & 0.721 & 0.256 & \\
\hline P94 & 43 & 0.712 & 0.251 & \\
\hline RVCD96 & 30 & 0.716 & 0.258 & 0.188 \\
\hline DS96 & 44 & 0.713 & 0.231 & \\
\hline $\mathrm{JCD}$ & 24 & 0.711 & 0.245 & $0.190^{*}$ \\
\hline$\overline{\text { Helioseismology }}$ & & $0.708-0.714$ & $0.238-0.259$ & $0.185-0.199$ \\
\hline
\end{tabular}

${ }^{*}$ private comunication by the authors 
TABLE IV. We present as a function of $x=R / R_{\odot}$ the helioseismological (best) values for $U=P / \rho\left(U_{\odot}\right)$, the relative partial uncertainties $(\delta U / U)_{k}$ corresponding to experimental errors $(\exp )$, model dependence $(\bmod )$, and to the parameters used in the inversion method $\left(\lambda, n_{F}, x_{f i t}\right.$ and $x_{o}$ ). Global errors $\Delta U / U$, estimated according to the statistical and the conservative approach, see Eqs. (10) and (11), are also shown.

\begin{tabular}{|c|c|c|c|c|c|c|c|c|c|}
\hline$x$ & $U_{\odot}$ [c.g.s.] & $\left(\frac{\delta U}{U}\right)_{e x p}$ & $\left(\frac{\delta U}{U}\right)_{\bmod }$ & $\left(\frac{\delta U}{U}\right)_{\lambda}$ & $\left(\frac{\delta U}{U}\right)_{n_{F}}$ & $\left(\frac{\delta U}{U}\right)_{x_{f}}$ & $\overline{\left(\frac{\delta U}{U}\right)_{x_{o}}}$ & $\left(\frac{\Delta U}{U}\right)_{s t a}$ & $\left(\frac{\Delta U}{U}\right)_{c o n}$ \\
\hline 4 & 15 & $6.829 \mathrm{E}-03$ & $1.170 \mathrm{E}-03$ & $\overline{80 E 0}$ & 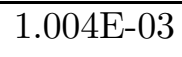 & $1.315 \mathrm{E}-02$ & $.591 \mathrm{E}-02$ & 1800 ח08 & \\
\hline & & $92 \mathrm{E}-03$ & & \pm & & 02 & & & \\
\hline 116 & $1.540 \mathrm{E}+15$ & $6.740 \mathrm{E}-03$ & $169 \mathrm{E}-03$ & $33 \mathrm{E}-04$ & $.027 \mathrm{E}-03$ & $1.269 \mathrm{E}-02$ & $1.571 \mathrm{E}-02$ & 067E-02 & -02 \\
\hline & $42 \mathrm{E}+15$ & $670 \mathrm{E}-03$ & 233 & 7OIL-04 & -03 & $1.233 \mathrm{E}-02$ & $555 \mathrm{E}-02$ & 02 & \\
\hline ) & $43 \mathrm{E}+15$ & $71 \mathrm{E}-03$ & $242 \mathrm{E}-$ & $126 \mathrm{E}-04$ & -03 & 1.183E-02 & $1.533 \mathrm{E}-02$ & -02 & \\
\hline & $44 \mathrm{E}+15$ & -03 & 3 & 2 & 3 & $1.116 \mathrm{E}-02$ & $1.503 \mathrm{E}-02$ & 03 & $4-$ \\
\hline 21 & & $6.257 \mathrm{E}-03$ & 3 & $t$ & -03 & $1.025 \mathrm{E}-02$ & E-02 & & \\
\hline 260 & $49 \mathrm{E}+15$ & $6.010 \mathrm{E}-03$ & $638 \mathrm{E}-03$ & $426 \mathrm{E}-\mathrm{C}$ & 219E-03 & $9.045 \mathrm{E}-03$ & $1.407 \mathrm{E}-02$ & E-03 & -02 \\
\hline-1 & $51 \mathrm{E}+15$ & $81 \mathrm{E}-03$ & $590 \mathrm{E}-03$ & 325 & $305 \mathrm{E}-03$ & 7.473E-03 & $1.332 \mathrm{E}-02$ & $216 \mathrm{E}-03$ & -02 \\
\hline & & $9 \mathrm{E}-03$ & 然 & די & 03 & $5.490 \mathrm{E}-03$ & $1.234 \mathrm{E}-02$ & & \\
\hline & & -03 & $48 \mathrm{E}$ & $\mid \varepsilon$ & 03 & $3.107 \mathrm{E}-03$ & $1.109 \mathrm{E}-02$ & -03 & $-U$ \\
\hline 482 & $1.560 \mathrm{E}$ & $4.026 \mathrm{E}-03$ & $346 \mathrm{E}-03$ & $72 \mathrm{E}-03$ & $46 \mathrm{E}-03$ & $5.675 \mathrm{E}-04$ & $9.564 \mathrm{E}-03$ & $344 \mathrm{E}-03$ & \\
\hline & $62 \mathrm{E}+15$ & & 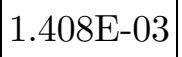 & & & -03 & -03 & 03 & \\
\hline 634 & $1.563 \mathrm{E}+15$ & $2.407 \mathrm{E}-03$ & $472 \mathrm{E}-03$ & 823E- ( & $.189 \mathrm{E}-03$ & $4.245 \mathrm{E}-03$ & $5.833 \mathrm{E}-03$ & $127 \mathrm{E}-03$ & $2-0$ \\
\hline & & E-03 & \pm 10 & $E-1$ & -03 & $5.052 \mathrm{E}-03$ & E-03 & -03 & \\
\hline & & & & 12 & & & & & -0 \\
\hline 0896 & $1.549 \mathrm{E}+15$ & $6.468 \mathrm{E}-04$ & $1.549 \mathrm{E}-03$ & $2.550 \mathrm{E}-03$ & $2.852 \mathrm{E}-03$ & $2.977 \mathrm{E}-03$ & $6.362 \mathrm{E}-04$ & $2.584 \mathrm{E}-03$ & $\mathrm{E}-0$ \\
\hline & $38 \mathrm{E}+15$ & 39E-04 & 1.496 & 495E- & -03 & E-03 & $1 \mathrm{E}-06$ & -03 & 0 \\
\hline 3 & $3 \mathrm{E}+15$ & -04 & $576 \mathrm{E}$ & 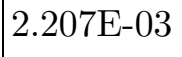 & -03 & 7.635E-04 & $0.000 \mathrm{E}+00$ & 3 & -0 \\
\hline 200 & $1.501 \mathrm{E}+15$ & $9.119 \mathrm{E}-04$ & $1.553 \mathrm{E}-03$ & $1.755 \mathrm{E}-03$ & $2.977 \mathrm{E}-03$ & 8.726E-05 & $0.000 \mathrm{E}+00$ & $1.949 \mathrm{E}-03$ & 7. \\
\hline & & 04 & & & & 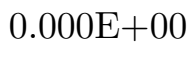 & +00 & & \\
\hline 40 & $8 \mathrm{E}+15$ & $8.597 \mathrm{E}-04$ & $313 \mathrm{E}-$ & E-1 & -5 & 0.00 & $0 \mathrm{E}+00$ & E-03 & $\mathrm{E}-0$ \\
\hline 75 & $1.394 \mathrm{E}+15$ & 7.009E-04 & $1.130 \mathrm{E}-03$ & 3.038E-( & $2.352 \mathrm{E}-0$ & $0.000 \mathrm{E}+00$ & $0.000 \mathrm{E}+00$ & $1.360 \mathrm{E}-03$ & E-03 \\
\hline 2 & $342 \mathrm{E}+15$ & -04 & 1.230 & & & & ק & 03 & 4. \\
\hline 380 & $1.283 \mathrm{E}+15$ & $18 \mathrm{E}-04$ & $313 \mathrm{E}-03$ & $9 \mathrm{E}-\mathrm{C}$ & $0 \mathrm{E}-\mathrm{C}$ & + & $0 \mathrm{E}+00$ & $9.900 \mathrm{E}-04$ & $\mathrm{E}-0$ \\
\hline 45 & $1.221 \mathrm{E}+15$ & 49E-04 & 3 & 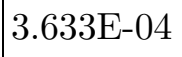 & 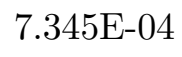 & $.000 \mathrm{E}+0$ & +00 & $821 \mathrm{E}-04$ & -0 \\
\hline 216 & $7 \mathrm{E}+15$ & $6.705 \mathrm{E}-04$ & $99 \mathrm{E}-03$ & 8. & & & F⿸尸 & & E-0 \\
\hline .2389 & $1.096 \mathrm{E}+15$ & $53 \mathrm{E}-04$ & $1.336 \mathrm{E}-03$ & 1 & 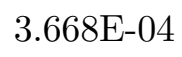 & $000 \mathrm{E}+00$ & $0 \mathrm{E}+00$ & $9.469 \mathrm{E}-04$ & E-03 \\
\hline 564 & $37 \mathrm{E}+15$ & 30E-04 & $415 \mathrm{E}-03$ & L-c & 4. & 0. & 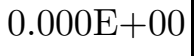 & $9.832 \mathrm{E}-04$ & -0 \\
\hline$=$ & +14 & $1 \mathrm{E}-04$ & P & 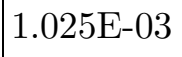 & & & & & E-0 \\
\hline .2927 & $286 \mathrm{E}+14$ & $5.502 \mathrm{E}-04$ & $1.606 \mathrm{~F}$ & 8 & $288 F_{1}$ & $0.000 \mathrm{E}+00$ & $0 \mathrm{E}+00$ & E-03 & E-03 \\
\hline 107 & $11 \mathrm{E}+14$ & $701 \mathrm{E}-04$ & $590 \mathrm{E}$ & $1^{\circ}$ & 1.5 & $0.0 \mathrm{c}-\mathrm{s}$ & $5+00$ & T-04 & $\mathrm{E}-0$ \\
\hline 3286 & $373 \mathrm{E}+14$ & 031E-04 & 402 & & & & c & -04 & E-0 \\
\hline .3466 & $958 \mathrm{E}+14$ & $4.220 \mathrm{E}-04$ & $1.380 \mathrm{E}-($ & 2.09 & $8.116 \mathrm{E}-0$ & 0.0 & $0 \mathrm{E}+00$ & $8.277 \mathrm{E}-04$ & $2.634 \mathrm{E}-0$ \\
\hline 46 & $564 \mathrm{E}+14$ & $4.168 \mathrm{E}-04$ & $.511 \mathrm{E}-03$ & 8.028 & E- & $\mathrm{E}+$ & $0 \mathrm{E}+00$ & $9.567 \mathrm{E}-04$ & $3.479 \mathrm{E}-03$ \\
\hline .3829 & $7.198 \mathrm{E}+14$ & $4.011 \mathrm{E}-04$ & $1.463 \mathrm{E}-03$ & 1.1 & 1 & $\Gamma+6>$ & $0.000 \mathrm{E}+00$ & $1.018 \mathrm{E}-03$ & $3.755 \mathrm{E}-03$ \\
\hline .4013 & $6.858 \mathrm{E}+14$ & $3.739 \mathrm{E}-04$ & $.456 \mathrm{E}-03$ & 1.45 & s & 0.0 & $\mathrm{E}+00$ & $9.570 \mathrm{E}-04$ & $3.497 \mathrm{E}-03$ \\
\hline .4198 & $535 \mathrm{E}+14$ & $830 \mathrm{E}-04$ & $.606 \mathrm{E}-03$ & 7.479E-04 & 1.007E-0: & $0.000 \mathrm{E}+00$ & $0.000 \mathrm{E}+00$ & $1.037 \mathrm{E}-03$ & $3.744 \mathrm{E}-03$ \\
\hline
\end{tabular}


\begin{tabular}{l|l|l|l|l|l|l|l|l|l|l|l|l|l|l}
0.4385 & $6.229 \mathrm{E}+14$ & $3.573 \mathrm{E}-04$ & $1.878 \mathrm{E}-03$ & $8.761 \mathrm{E}-04$ & $9.760 \mathrm{E}-04$ & $0.000 \mathrm{E}+00$ & $0.000 \mathrm{E}+00$ & $1.159 \mathrm{E}-03$ & $4.087 \mathrm{E}-03$
\end{tabular} $\begin{array}{lllllllllll}0.4575 & 5.942 \mathrm{E}+14 & 3.248 \mathrm{E}-04 & 2.010 \mathrm{E}-03 & 5.610 \mathrm{E}-04 & 9.858 \mathrm{E}-04 & 0.000 \mathrm{E}+00 & 0.000 \mathrm{E}+00 & 1.165 \mathrm{E}-03 & 3.882 \mathrm{E}-03\end{array}$ \begin{tabular}{lll|llllllllll}
0.4768 & $5.670 \mathrm{E}+14$ & $3.628 \mathrm{E}-04$ & $2.076 \mathrm{E}-03$ & $3.094 \mathrm{E}-04$ & $1.107 \mathrm{E}-03$ & $0.000 \mathrm{E}+00$ & $0.000 \mathrm{E}+00$ & $1.200 \mathrm{E}-03$ & $3.856 \mathrm{E}-03$
\end{tabular} $\begin{array}{lllllllllll}0.4963 & 5.404 \mathrm{E}+14 & 2.796 \mathrm{E}-04 & 1.966 \mathrm{E}-03 & 1.122 \mathrm{E}-03 & 1.106 \mathrm{E}-03 & 0.000 \mathrm{E}+00 & 0.000 \mathrm{E}+00 & 1.268 \mathrm{E}-03 & 4.474 \mathrm{E}-03\end{array}$ $\begin{array}{lllllllllll}0.5160 & 5.154 \mathrm{E}+14 & 2.913 \mathrm{E}-04 & 1.775 \mathrm{E}-03 & 1.169 \mathrm{E}-03 & 1.070 \mathrm{E}-03 & 0.000 \mathrm{E}+00 & 0.000 \mathrm{E}+00 & 1.199 \mathrm{E}-03 & 4.305 \mathrm{E}-03\end{array}$ $\begin{array}{llllllllllll}0.5360 & 4.916 \mathrm{E}+14 & 2.744 \mathrm{E}-04 & 1.787 \mathrm{E}-03 & 8.637 \mathrm{E}-04 & 1.140 \mathrm{E}-03 & 0.000 \mathrm{E}+00 & 0.000 \mathrm{E}+00 & 1.153 \mathrm{E}-03 & 4.065 \mathrm{E}-03\end{array}$ $\begin{array}{lllllllllll}0.5562 & 4.686 \mathrm{E}+14 & 2.798 \mathrm{E}-04 & 1.987 \mathrm{E}-03 & 6.411 \mathrm{E}-04 & 1.199 \mathrm{E}-03 & 0.000 \mathrm{E}+00 & 0.000 \mathrm{E}+00 & 1.212 \mathrm{E}-03 & 4.106 \mathrm{E}-03\end{array}$ $\begin{array}{llllllllllll}0.5765 & 4.464 \mathrm{E}+14 & 3.116 \mathrm{E}-04 & 1.916 \mathrm{E}-03 & 4.761 \mathrm{E}-04 & 1.179 \mathrm{E}-03 & 0.000 \mathrm{E}+00 & 0.000 \mathrm{E}+00 & 1.160 \mathrm{E}-03 & 3.882 \mathrm{E}-03\end{array}$ $\begin{array}{lllllllllll}0.5968 & 4.248 \mathrm{E}+14 & 2.691 \mathrm{E}-04 & 1.953 \mathrm{E}-03 & 9.881 \mathrm{E}-04 & 1.298 \mathrm{E}-03 & 0.000 \mathrm{E}+00 & 0.000 \mathrm{E}+00 & 1.279 \mathrm{E}-03 & 4.508 \mathrm{E}-03\end{array}$ $\begin{array}{llllllllllll}0.6172 & 4.040 \mathrm{E}+14 & 2.478 \mathrm{E}-04 & 1.544 \mathrm{E}-03 & 1.260 \mathrm{E}-03 & 1.238 \mathrm{E}-03 & 0.000 \mathrm{E}+00 & 0.000 \mathrm{E}+00 & 1.180 \mathrm{E}-03 & 4.290 \mathrm{E}-03\end{array}$ $\begin{array}{llllllllllll}0.6374 & 3.837 \mathrm{E}+14 & 2.150 \mathrm{E}-04 & 1.871 \mathrm{E}-03 & 1.497 \mathrm{E}-03 & 1.288 \mathrm{E}-03 & 0.000 \mathrm{E}+00 & 0.000 \mathrm{E}+00 & 1.365 \mathrm{E}-03 & 4.872 \mathrm{E}-03\end{array}$ $\begin{array}{llllllllllll}0.6574 & 3.638 \mathrm{E}+14 & 2.108 \mathrm{E}-04 & 1.861 \mathrm{E}-03 & 8.875 \mathrm{E}-04 & 1.423 \mathrm{E}-03 & 0.000 \mathrm{E}+00 & 0.000 \mathrm{E}+00 & 1.257 \mathrm{E}-03 & 4.383 \mathrm{E}-03\end{array}$ $\begin{array}{llllllllllll}0.6769 & 3.435 \mathrm{E}+14 & 2.547 \mathrm{E}-04 & 1.593 \mathrm{E}-03 & 1.351 \mathrm{E}-04 & 1.479 \mathrm{E}-03 & 0.000 \mathrm{E}+00 & 0.000 \mathrm{E}+00 & 1.096 \mathrm{E}-03 & 3.462 \mathrm{E}-03\end{array}$ $\begin{array}{lllllllllll}0.6957 & 3.218 \mathrm{E}+14 & 2.014 \mathrm{E}-04 & 2.392 \mathrm{E}-03 & 1.294 \mathrm{E}-03 & 1.622 \mathrm{E}-03 & 0.000 \mathrm{E}+00 & 0.000 \mathrm{E}+00 & 1.586 \mathrm{E}-03 & 5.508 \mathrm{E}-03\end{array}$ $\begin{array}{lllllllllll}0.7131 & 2.991 \mathrm{E}+14 & 1.749 \mathrm{E}-04 & 2.170 \mathrm{E}-03 & 1.990 \mathrm{E}-03 & 1.821 \mathrm{E}-03 & 0.000 \mathrm{E}+00 & 0.000 \mathrm{E}+00 & 1.733 \mathrm{E}-03 & 6.155 \mathrm{E}-03\end{array}$ \begin{tabular}{ll|lllllllll}
0.7298 & $2.751 \mathrm{E}+14$ & $2.010 \mathrm{E}-04$ & $2.095 \mathrm{E}-03$ & $1.692 \mathrm{E}-03$ & $2.014 \mathrm{E}-03$ & $0.000 \mathrm{E}+00$ & $0.000 \mathrm{E}+00$ & $1.684 \mathrm{E}-03$ & $6.002 \mathrm{E}-03$
\end{tabular} $\begin{array}{lllllllllll}0.7461 & 2.529 \mathrm{E}+14 & 1.657 \mathrm{E}-04 & 2.068 \mathrm{E}-03 & 1.216 \mathrm{E}-03 & 2.155 \mathrm{E}-03 & 0.000 \mathrm{E}+00 & 0.000 \mathrm{E}+00 & 1.614 \mathrm{E}-03 & 5.604 \mathrm{E}-03\end{array}$ $\begin{array}{lllllllllll}0.7619 & 2.321 \mathrm{E}+14 & 1.512 \mathrm{E}-04 & 2.196 \mathrm{E}-03 & 1.448 \mathrm{E}-03 & 2.272 \mathrm{E}-03 & 0.000 \mathrm{E}+00 & 0.000 \mathrm{E}+00 & 1.740 \mathrm{E}-03 & 6.067 \mathrm{E}-03\end{array}$ $\begin{array}{lllllllllllll}0.7772 & 2.127 \mathrm{E}+14 & 1.886 \mathrm{E}-04 & 2.334 \mathrm{E}-03 & 1.872 \mathrm{E}-03 & 2.344 \mathrm{E}-03 & 0.000 \mathrm{E}+00 & 0.000 \mathrm{E}+00 & 1.903 \mathrm{E}-03 & 6.738 \mathrm{E}-03\end{array}$ $\begin{array}{lllllllllll}0.7920 & 1.946 \mathrm{E}+14 & 1.557 \mathrm{E}-04 & 2.381 \mathrm{E}-03 & 1.845 \mathrm{E}-03 & 2.390 \mathrm{E}-03 & 0.000 \mathrm{E}+00 & 0.000 \mathrm{E}+00 & 1.924 \mathrm{E}-03 & 6.771 \mathrm{E}-03\end{array}$ $\begin{array}{lllllllllll}0.8063 & 1.779 \mathrm{E}+14 & 1.642 \mathrm{E}-04 & 2.641 \mathrm{E}-03 & 1.843 \mathrm{E}-03 & 2.575 \mathrm{E}-03 & 0.000 \mathrm{E}+00 & 0.000 \mathrm{E}+00 & 2.063 \mathrm{E}-03 & 7.224 \mathrm{E}-03\end{array}$ $\begin{array}{llllllllllll}0.8199 & 1.623 \mathrm{E}+14 & 1.664 \mathrm{E}-04 & 3.086 \mathrm{E}-03 & 2.035 \mathrm{E}-03 & 2.850 \mathrm{E}-03 & 0.000 \mathrm{E}+00 & 0.000 \mathrm{E}+00 & 2.335 \mathrm{E}-03 & 8.137 \mathrm{E}-03\end{array}$ $\begin{array}{llllllllllll}0.8329 & 1.478 \mathrm{E}+14 & 1.779 \mathrm{E}-04 & 3.271 \mathrm{E}-03 & 2.179 \mathrm{E}-03 & 2.969 \mathrm{E}-03 & 0.000 \mathrm{E}+00 & 0.000 \mathrm{E}+00 & 2.464 \mathrm{E}-03 & 8.597 \mathrm{E}-03\end{array}$ $\begin{array}{lllllllllll}0.8453 & 1.345 \mathrm{E}+14 & 1.844 \mathrm{E}-04 & 3.385 \mathrm{E}-03 & 2.268 \mathrm{E}-03 & 3.035 \mathrm{E}-03 & 0.000 \mathrm{E}+00 & 0.000 \mathrm{E}+00 & 2.542 \mathrm{E}-03 & 8.872 \mathrm{E}-03\end{array}$ $\begin{array}{lllllllllll}0.8571 & 1.222 \mathrm{E}+14 & 1.939 \mathrm{E}-04 & 3.700 \mathrm{E}-03 & 2.426 \mathrm{E}-03 & 3.281 \mathrm{E}-03 & 0.000 \mathrm{E}+00 & 0.000 \mathrm{E}+00 & 2.756 \mathrm{E}-03 & 9.600 \mathrm{E}-03\end{array}$ $\begin{array}{llllllllllll}0.8682 & 1.109 \mathrm{E}+14 & 1.957 \mathrm{E}-04 & 4.087 \mathrm{E}-03 & 2.643 \mathrm{E}-03 & 3.659 \mathrm{E}-03 & 0.000 \mathrm{E}+00 & 0.000 \mathrm{E}+00 & 3.046 \mathrm{E}-03 & 1.058 \mathrm{E}-02\end{array}$ $\begin{array}{lllllllllll}0.8786 & 1.005 \mathrm{E}+14 & 2.100 \mathrm{E}-04 & 4.209 \mathrm{E}-03 & 2.839 \mathrm{E}-03 & 3.945 \mathrm{E}-03 & 0.000 \mathrm{E}+00 & 0.000 \mathrm{E}+00 & 3.217 \mathrm{E}-03 & 1.120 \mathrm{E}-02\end{array}$ $\begin{array}{lllllllllll}0.8884 & 9.099 \mathrm{E}+13 & 2.245 \mathrm{E}-04 & 4.166 \mathrm{E}-03 & 3.020 \mathrm{E}-03 & 4.157 \mathrm{E}-03 & 0.000 \mathrm{E}+00 & 0.000 \mathrm{E}+00 & 3.309 \mathrm{E}-03 & 1.157 \mathrm{E}-02\end{array}$ $\begin{array}{lllllllllll}0.8975 & 8.229 \mathrm{E}+13 & 2.475 \mathrm{E}-04 & 3.969 \mathrm{E}-03 & 3.261 \mathrm{E}-03 & 4.460 \mathrm{E}-03 & 0.000 \mathrm{E}+00 & 0.000 \mathrm{E}+00 & 3.404 \mathrm{E}-03 & 1.194 \mathrm{E}-02\end{array}$ $\begin{array}{lllllllllll}0.9060 & 7.435 \mathrm{E}+13 & 2.748 \mathrm{E}-04 & 3.865 \mathrm{E}-03 & 3.611 \mathrm{E}-03 & 4.958 \mathrm{E}-03 & 0.000 \mathrm{E}+00 & 0.000 \mathrm{E}+00 & 3.628 \mathrm{E}-03 & 1.271 \mathrm{E}-02\end{array}$ $\begin{array}{llllllllllll}0.9139 & 6.712 \mathrm{E}+13 & 3.047 \mathrm{E}-04 & 3.810 \mathrm{E}-03 & 4.032 \mathrm{E}-03 & 5.591 \mathrm{E}-03 & 0.000 \mathrm{E}+00 & 0.000 \mathrm{E}+00 & 3.941 \mathrm{E}-03 & 1.374 \mathrm{E}-02\end{array}$ $\begin{array}{lllllllllll}0.9211 & 6.054 \mathrm{E}+13 & 3.342 \mathrm{E}-04 & 3.663 \mathrm{E}-03 & 4.463 \mathrm{E}-03 & 6.215 \mathrm{E}-03 & 0.000 \mathrm{E}+00 & 0.000 \mathrm{E}+00 & 4.245 \mathrm{E}-03 & 1.467 \mathrm{E}-02\end{array}$ $\begin{array}{llllllllllll}0.9278 & 5.457 \mathrm{E}+13 & 3.595 \mathrm{E}-04 & 3.464 \mathrm{E}-03 & 4.866 \mathrm{E}-03 & 6.722 \mathrm{E}-03 & 0.000 \mathrm{E}+00 & 0.000 \mathrm{E}+00 & 4.500 \mathrm{E}-03 & 1.541 \mathrm{E}-02\end{array}$ $\begin{array}{lllllllllll}0.9340 & 4.915 \mathrm{E}+13 & 3.843 \mathrm{E}-04 & 3.496 \mathrm{E}-03 & 5.239 \mathrm{E}-03 & 7.195 \mathrm{E}-03 & 0.000 \mathrm{E}+00 & 0.000 \mathrm{E}+00 & 4.785 \mathrm{E}-03 & 1.631 \mathrm{E}-02\end{array}$

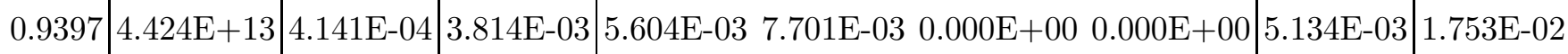
$\begin{array}{llllllllllll}0.9449 & 3.980 \mathrm{E}+13 & 4.487 \mathrm{E}-04 & 4.083 \mathrm{E}-03 & 5.977 \mathrm{E}-03 & 8.294 \mathrm{E}-03 & 0.000 \mathrm{E}+00 & 0.000 \mathrm{E}+00 & 5.509 \mathrm{E}-03 & 1.880 \mathrm{E}-02\end{array}$ $\begin{array}{llllllllllll}0.9496 & 3.578 \mathrm{E}+13 & 4.883 \mathrm{E}-04 & 4.579 \mathrm{E}-03 & 6.364 \mathrm{E}-03 & 8.998 \mathrm{E}-03 & 0.000 \mathrm{E}+00 & 0.000 \mathrm{E}+00 & 5.972 \mathrm{E}-03 & 2.043 \mathrm{E}-02\end{array}$ $\begin{array}{lllllllllll}0.9540 & 3.215 \mathrm{E}+13 & 5.357 \mathrm{E}-04 & 5.507 \mathrm{E}-03 & 6.788 \mathrm{E}-03 & 9.830 \mathrm{E}-03 & 0.000 \mathrm{E}+00 & 0.000 \mathrm{E}+00 & 6.583 \mathrm{E}-03 & 2.266 \mathrm{E}-02\end{array}$ $\begin{array}{lllllllllll}0.9579 & 2.887 \mathrm{E}+13 & 5.969 \mathrm{E}-04 & 6.528 \mathrm{E}-03 & 7.357 \mathrm{E}-03 & 1.085 \mathrm{E}-02 & 0.000 \mathrm{E}+00 & 0.000 \mathrm{E}+00 & 7.328 \mathrm{E}-03 & 2.533 \mathrm{E}-02\end{array}$ $\begin{array}{lllllllllll}0.9616 & 2.591 \mathrm{E}+13 & 6.713 \mathrm{E}-04 & 7.725 \mathrm{E}-03 & 8.151 \mathrm{E}-03 & 1.212 \mathrm{E}-02 & 0.000 \mathrm{E}+00 & 0.000 \mathrm{E}+00 & 8.269 \mathrm{E}-03 & 2.867 \mathrm{E}-02\end{array}$ \begin{tabular}{ll|lllllllll}
0.9649 & $2.323 \mathrm{E}+13$ & $7.554 \mathrm{E}-04$ & $9.347 \mathrm{E}-03$ & $9.197 \mathrm{E}-03$ & $1.365 \mathrm{E}-02$ & $0.000 \mathrm{E}+00$ & $0.000 \mathrm{E}+00$ & $9.473 \mathrm{E}-03$ & $3.295 \mathrm{E}-02$
\end{tabular} $\begin{array}{lllllllllll}0.9679 & 2.083 \mathrm{E}+13 & 8.474 \mathrm{E}-04 & 1.094 \mathrm{E}-02 & 1.049 \mathrm{E}-02 & 1.539 \mathrm{E}-02 & 0.000 \mathrm{E}+00 & 0.000 \mathrm{E}+00 & 1.081 \mathrm{E}-02 & 3.767 \mathrm{E}-02\end{array}$ $\begin{array}{lllllllllll}0.9706 & 1.867 \mathrm{E}+13 & 9.464 \mathrm{E}-04 & 1.263 \mathrm{E}-02 & 1.202 \mathrm{E}-02 & 1.729 \mathrm{E}-02 & 0.000 \mathrm{E}+00 & 0.000 \mathrm{E}+00 & 1.229 \mathrm{E}-02 & 4.289 \mathrm{E}-02\end{array}$

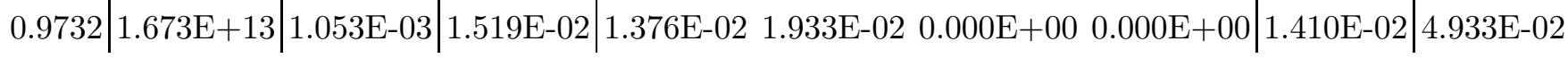


\begin{tabular}{l|l|l|l|l|l|l|l|l|l|l|l|l|l|l|}
0.9755 & $1.499 \mathrm{E}+13$ & $1.168 \mathrm{E}-03$ & $1.821 \mathrm{E}-02$ & $1.565 \mathrm{E}-02$ & $2.148 \mathrm{E}-02$ & $0.000 \mathrm{E}+00$ & $0.000 \mathrm{E}+00$ & $1.612 \mathrm{E}-02$ & $5.651 \mathrm{E}-02$
\end{tabular} $\begin{array}{llllllllll}0.9776 & 1.344 \mathrm{E}+13 & 1.291 \mathrm{E}-03 & 2.145 \mathrm{E}-02 & 1.766 \mathrm{E}-02 & 2.368 \mathrm{E}-02 & 0.000 \mathrm{E}+00 & 0.000 \mathrm{E}+00 & 1.826 \mathrm{E}-02 & 6.408 \mathrm{E}-02\end{array}$ $\begin{array}{lllllllllll}0.9795 & 1.204 \mathrm{E}+13 & 1.416 \mathrm{E}-03 & 2.568 \mathrm{E}-02 & 1.968 \mathrm{E}-02 & 2.584 \mathrm{E}-02 & 0.000 \mathrm{E}+00 & 0.000 \mathrm{E}+00 & 2.072 \mathrm{E}-02 & 7.262 \mathrm{E}-02\end{array}$ \begin{tabular}{ll|llllllll}
0.9812 & $1.079 \mathrm{E}+13$ & $1.541 \mathrm{E}-03$ & $3.015 \mathrm{E}-02$ & $2.166 \mathrm{E}-02$ & $2.791 \mathrm{E}-02$ & $0.000 \mathrm{E}+00$ & $0.000 \mathrm{E}+00$ & $2.323 \mathrm{E}-02$ & $8.126 \mathrm{E}-02$
\end{tabular} $\begin{array}{lllllllllll}0.9828 & 9.674 \mathrm{E}+12 & 1.663 \mathrm{E}-03 & 3.416 \mathrm{E}-02 & 2.353 \mathrm{E}-02 & 2.985 \mathrm{E}-02 & 0.000 \mathrm{E}+00 & 0.000 \mathrm{E}+00 & 2.557 \mathrm{E}-02 & 8.921 \mathrm{E}-02\end{array}$ $\begin{array}{lllllllllll}0.9842 & 8.665 \mathrm{E}+12 & 1.779 \mathrm{E}-03 & 3.766 \mathrm{E}-02 & 2.529 \mathrm{E}-02 & 3.165 \mathrm{E}-02 & 0.000 \mathrm{E}+00 & 0.000 \mathrm{E}+00 & 2.767 \mathrm{E}-02 & 9.637 \mathrm{E}-02\end{array}$ $\begin{array}{lllllllllll}0.9854 & 7.757 \mathrm{E}+12 & 1.889 \mathrm{E}-03 & 4.018 \mathrm{E}-02 & 2.693 \mathrm{E}-02 & 3.331 \mathrm{E}-02 & 0.000 \mathrm{E}+00 & 0.000 \mathrm{E}+00 & 2.938 \mathrm{E}-02 & 1.023 \mathrm{E}-01\end{array}$ $\begin{array}{llllllllllll}0.9866 & 6.943 \mathrm{E}+12 & 1.993 \mathrm{E}-03 & 4.193 \mathrm{E}-02 & 2.845 \mathrm{E}-02 & 3.484 \mathrm{E}-02 & 0.000 \mathrm{E}+00 & 0.000 \mathrm{E}+00 & 3.076 \mathrm{E}-02 & 1.072 \mathrm{E}-01\end{array}$ \begin{tabular}{ll|lllllllll}
0.9876 & $6.214 \mathrm{E}+12$ & $2.092 \mathrm{E}-03$ & $4.315 \mathrm{E}-02$ & $2.988 \mathrm{E}-02$ & $3.625 \mathrm{E}-02$ & $0.000 \mathrm{E}+00$ & $0.000 \mathrm{E}+00$ & $3.191 \mathrm{E}-02$ & $1.114 \mathrm{E}-01$
\end{tabular} \begin{tabular}{ll|lllllllll}
0.9886 & $5.566 \mathrm{E}+12$ & $2.185 \mathrm{E}-03$ & $4.406 \mathrm{E}-02$ & $3.122 \mathrm{E}-02$ & $3.757 \mathrm{E}-02$ & $0.000 \mathrm{E}+00$ & $0.000 \mathrm{E}+00$ & $3.291 \mathrm{E}-02$ & $1.150 \mathrm{E}-01$
\end{tabular} $\begin{array}{llllllllllll}0.9895 & 4.991 \mathrm{E}+12 & 2.273 \mathrm{E}-03 & 4.498 \mathrm{E}-02 & 3.248 \mathrm{E}-02 & 3.881 \mathrm{E}-02 & 0.000 \mathrm{E}+00 & 0.000 \mathrm{E}+00 & 3.387 \mathrm{E}-02 & 1.185 \mathrm{E}-01\end{array}$ $\begin{array}{llllllllllll}0.9903 & 4.482 \mathrm{E}+12 & 2.357 \mathrm{E}-03 & 4.627 \mathrm{E}-02 & 3.366 \mathrm{E}-02 & 3.996 \mathrm{E}-02 & 0.000 \mathrm{E}+00 & 0.000 \mathrm{E}+00 & 3.492 \mathrm{E}-02 & 1.222 \mathrm{E}-01\end{array}$ $\begin{array}{lllllllllll}0.9911 & 4.033 \mathrm{E}+12 & 2.435 \mathrm{E}-03 & 4.798 \mathrm{E}-02 & 3.478 \mathrm{E}-02 & 4.103 \mathrm{E}-02 & 0.000 \mathrm{E}+00 & 0.000 \mathrm{E}+00 & 3.606 \mathrm{E}-02 & 1.262 \mathrm{E}-01\end{array}$ \begin{tabular}{ll|l|l|lllllll}
0.9918 & $3.639 \mathrm{E}+12$ & $2.510 \mathrm{E}-03$ & $4.943 \mathrm{E}-02$ & $3.582 \mathrm{E}-02$ & $4.203 \mathrm{E}-02$ & $0.000 \mathrm{E}+00$ & $0.000 \mathrm{E}+00$ & $3.708 \mathrm{E}-02$ & $1.298 \mathrm{E}-01$
\end{tabular} $\begin{array}{lllllllllll}0.9925 & 3.290 \mathrm{E}+12 & 2.579 \mathrm{E}-03 & 5.046 \mathrm{E}-02 & 3.679 \mathrm{E}-02 & 4.296 \mathrm{E}-02 & 0.000 \mathrm{E}+00 & 0.000 \mathrm{E}+00 & 3.792 \mathrm{E}-02 & 1.328 \mathrm{E}-01\end{array}$ \begin{tabular}{l|l|l|l|lllllll}
0.9931 & $2.981 \mathrm{E}+12$ & $2.644 \mathrm{E}-03$ & $5.045 \mathrm{E}-02$ & $3.769 \mathrm{E}-02$ & $4.381 \mathrm{E}-02$ & $0.000 \mathrm{E}+00$ & $0.000 \mathrm{E}+00$ & $3.838 \mathrm{E}-02$ & $1.346 \mathrm{E}-01$
\end{tabular} \begin{tabular}{ll|l|l|lllllll}
0.9937 & $2.708 \mathrm{E}+12$ & $2.705 \mathrm{E}-03$ & $4.942 \mathrm{E}-02$ & $3.853 \mathrm{E}-02$ & $4.460 \mathrm{E}-02$ & $0.000 \mathrm{E}+00$ & $0.000 \mathrm{E}+00$ & $3.848 \mathrm{E}-02$ & $1.353 \mathrm{E}-01$
\end{tabular} \begin{tabular}{ll|l|l|llllll}
0.9942 & $2.465 \mathrm{E}+12$ & $2.761 \mathrm{E}-03$ & $4.791 \mathrm{E}-02$ & $3.931 \mathrm{E}-02$ & $4.534 \mathrm{E}-02$ & $0.000 \mathrm{E}+00$ & $0.000 \mathrm{E}+00$ & $3.842 \mathrm{E}-02$ & $1.353 \mathrm{E}-01$
\end{tabular}

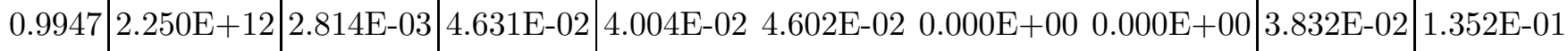
\begin{tabular}{ll|l|l|lllllll}
0.9952 & $2.059 \mathrm{E}+12$ & $2.863 \mathrm{E}-03$ & $4.491 \mathrm{E}-02$ & $4.071 \mathrm{E}-02$ & $4.664 \mathrm{E}-02$ & $0.000 \mathrm{E}+00$ & $0.000 \mathrm{E}+00$ & $3.827 \mathrm{E}-02$ & $1.351 \mathrm{E}-01$
\end{tabular}

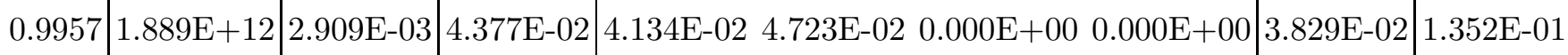

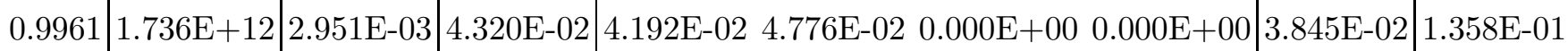

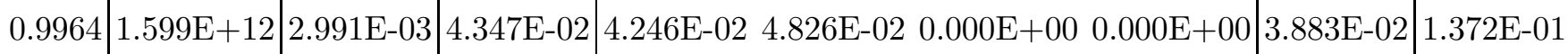
$\begin{array}{llllllllllll}0.9968 & 1.474 \mathrm{E}+12 & 3.028 \mathrm{E}-03 & 4.475 \mathrm{E}-02 & 4.297 \mathrm{E}-02 & 4.872 \mathrm{E}-02 & 0.000 \mathrm{E}+00 & 0.000 \mathrm{E}+00 & 3.947 \mathrm{E}-02 & 1.395 \mathrm{E}-01\end{array}$ \begin{tabular}{ll|llllllllll}
0.9971 & $1.360 \mathrm{E}+12$ & $3.062 \mathrm{E}-03$ & $4.750 \mathrm{E}-02$ & $4.343 \mathrm{E}-02$ & $4.914 \mathrm{E}-02$ & $0.000 \mathrm{E}+00$ & $0.000 \mathrm{E}+00$ & $4.052 \mathrm{E}-02$ & $1.431 \mathrm{E}-01$
\end{tabular} $\begin{array}{lllllllllll}0.9974 & 1.256 \mathrm{E}+12 & 3.094 \mathrm{E}-03 & 5.212 \mathrm{E}-02 & 4.386 \mathrm{E}-02 & 4.953 \mathrm{E}-02 & 0.000 \mathrm{E}+00 & 0.000 \mathrm{E}+00 & 4.214 \mathrm{E}-02 & 1.486 \mathrm{E}-01\end{array}$ \begin{tabular}{ll|l|l|lllllll}
0.9977 & $1.160 \mathrm{E}+12$ & $3.123 \mathrm{E}-03$ & $5.917 \mathrm{E}-02$ & $4.426 \mathrm{E}-02$ & $4.989 \mathrm{E}-02$ & $0.000 \mathrm{E}+00$ & $0.000 \mathrm{E}+00$ & $4.461 \mathrm{E}-02$ & $1.564 \mathrm{E}-01$
\end{tabular} $\begin{array}{llllllllllll}0.9980 & 1.070 \mathrm{E}+12 & 3.150 \mathrm{E}-03 & 6.888 \mathrm{E}-02 & 4.462 \mathrm{E}-02 & 5.022 \mathrm{E}-02 & 0.000 \mathrm{E}+00 & 0.000 \mathrm{E}+00 & 4.813 \mathrm{E}-02 & 1.669 \mathrm{E}-01\end{array}$ \begin{tabular}{ll|llllllllll}
0.9982 & $9.852 \mathrm{E}+11$ & $3.175 \mathrm{E}-03$ & $8.197 \mathrm{E}-02$ & $4.496 \mathrm{E}-02$ & $5.052 \mathrm{E}-02$ & $0.000 \mathrm{E}+00$ & $0.000 \mathrm{E}+00$ & $5.316 \mathrm{E}-02$ & $1.806 \mathrm{E}-01$
\end{tabular} \begin{tabular}{ll|lllllllll}
0.9984 & $9.046 \mathrm{E}+11$ & $3.197 \mathrm{E}-03$ & $9.911 \mathrm{E}-02$ & $4.526 \mathrm{E}-02$ & $5.079 \mathrm{E}-02$ & $0.000 \mathrm{E}+00$ & $0.000 \mathrm{E}+00$ & $6.013 \mathrm{E}-02$ & $1.984 \mathrm{E}-01$
\end{tabular} \begin{tabular}{ll|l|l|lllllll}
0.9986 & $8.266 \mathrm{E}+11$ & $3.217 \mathrm{E}-03$ & $1.204 \mathrm{E}-01$ & $4.554 \mathrm{E}-02$ & $5.104 \mathrm{E}-02$ & $0.000 \mathrm{E}+00$ & $0.000 \mathrm{E}+00$ & $6.926 \mathrm{E}-02$ & $2.202 \mathrm{E}-01$
\end{tabular} $\begin{array}{llllllllllll}0.9988 & 7.502 \mathrm{E}+11 & 3.235 \mathrm{E}-03 & 1.452 \mathrm{E}-01 & 4.578 \mathrm{E}-02 & 5.125 \mathrm{E}-02 & 0.000 \mathrm{E}+00 & 0.000 \mathrm{E}+00 & 8.032 \mathrm{E}-02 & 2.454 \mathrm{E}-01\end{array}$ $\begin{array}{llllllllllll}0.9989 & 6.751 \mathrm{E}+11 & 3.250 \mathrm{E}-03 & 1.720 \mathrm{E}-01 & 4.598 \mathrm{E}-02 & 5.143 \mathrm{E}-02 & 0.000 \mathrm{E}+00 & 0.000 \mathrm{E}+00 & 9.269 \mathrm{E}-02 & 2.727 \mathrm{E}-01\end{array}$ \begin{tabular}{ll|llllllllll}
0.9990 & $6.019 \mathrm{E}+11$ & $3.262 \mathrm{E}-03$ & $2.003 \mathrm{E}-01$ & $4.614 \mathrm{E}-02$ & $5.158 \mathrm{E}-02$ & $0.000 \mathrm{E}+00$ & $0.000 \mathrm{E}+00$ & $1.060 \mathrm{E}-01$ & $3.013 \mathrm{E}-01$
\end{tabular} \begin{tabular}{ll|lllllllll}
0.9991 & $5.340 \mathrm{E}+11$ & $3.270 \mathrm{E}-03$ & $2.295 \mathrm{E}-01$ & $4.625 \mathrm{E}-02$ & $5.167 \mathrm{E}-02$ & $0.000 \mathrm{E}+00$ & $0.000 \mathrm{E}+00$ & $1.199 \mathrm{E}-01$ & $3.307 \mathrm{E}-01$
\end{tabular} \begin{tabular}{ll|llllllllll}
0.9992 & $4.739 \mathrm{E}+11$ & $3.275 \mathrm{E}-03$ & $1.744 \mathrm{E}-01$ & $4.632 \mathrm{E}-02$ & $5.173 \mathrm{E}-02$ & $0.000 \mathrm{E}+00$ & $0.000 \mathrm{E}+00$ & $9.389 \mathrm{E}-02$ & $2.758 \mathrm{E}-01$
\end{tabular} $\begin{array}{llllllllllll}0.9992 & 4.239 \mathrm{E}+11 & 3.279 \mathrm{E}-03 & 4.945 \mathrm{E}-02 & 4.637 \mathrm{E}-02 & 5.178 \mathrm{E}-02 & 0.000 \mathrm{E}+00 & 0.000 \mathrm{E}+00 & 4.268 \mathrm{E}-02 & 1.509 \mathrm{E}-01\end{array}$ $\begin{array}{lllllllllllll}0.9992 & 3.881 \mathrm{E}+11 & 3.283 \mathrm{E}-03 & 4.003 \mathrm{E}-02 & 4.642 \mathrm{E}-02 & 5.182 \mathrm{E}-02 & 0.000 \mathrm{E}+00 & 0.000 \mathrm{E}+00 & 4.017 \mathrm{E}-02 & 1.416 \mathrm{E}-01\end{array}$

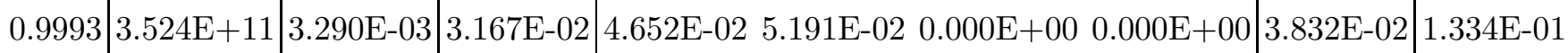
$\begin{array}{llllllllllll}0.9995 & 3.067 \mathrm{E}+11 & 3.310 \mathrm{E}-03 & 2.935 \mathrm{E}-02 & 4.679 \mathrm{E}-02 & 5.215 \mathrm{E}-02 & 0.000 \mathrm{E}+00 & 0.000 \mathrm{E}+00 & 3.802 \mathrm{E}-02 & 1.316 \mathrm{E}-01\end{array}$

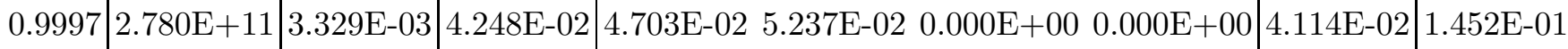
\begin{tabular}{l|l|l|l|l|l|l|l|l|l|l}
0.9999 & $2.713 \mathrm{E}+11$ & $3.346 \mathrm{E}-03$ & $2.949 \mathrm{E}-02$ & $4.727 \mathrm{E}-02$ & $5.257 \mathrm{E}-02$ & $0.000 \mathrm{E}+00$ & $0.000 \mathrm{E}+00$ & $3.834 \mathrm{E}-02$ & $1.327 \mathrm{E}-01$ \\
\hline
\end{tabular} 


\section{FIGURES}

FIG. 1. Helioseismological determinations of the photospheric Helium abundance, $Y_{p h}$. Same notation as in Table III. The allowed region according to the present work is between the dashed lines.

FIG. 2. The estimated global relative uncertainty on $U=P / \rho$, according to the conservative approach, Eq. (11) (thick line), and to the statistical approach, Eq. (10) (thin line).

FIG. 3. Helioseismological determinations and solar model predictions of properties of the outer sun. The box defines the region allowed by helioseismology. Open circles denote models without diffusion, squares models with He diffusion, full circles models with He and heavier elements diffusion.

FIG. 4. The difference between $U$ as predicted by selected solar models, $U_{\text {mod }}$ and the helioseismological determination, $U_{\odot}$, normalized to this latter. The allowed region is that within the

thick lines, corresponding to $\left(\frac{\Delta U}{U}\right)_{c o n}$. SUN24 is the "model 0" of Ref. [23]; FRANEC96 is the "best" model with He and heavier elements diffusion of Ref. [35]; BP95 is the model with metal and He diffusion of Ref. 34]; JCD is the "model S" of Ref. 24].

FIG. 5. a) The profiles of $U$ according to FRANEC96 and FRANEC96-OLD (full and dashed lines, respectively). b) The relative differences $\Delta k / k$ between 19 - and 12- elements opacities, along the solar profile. 


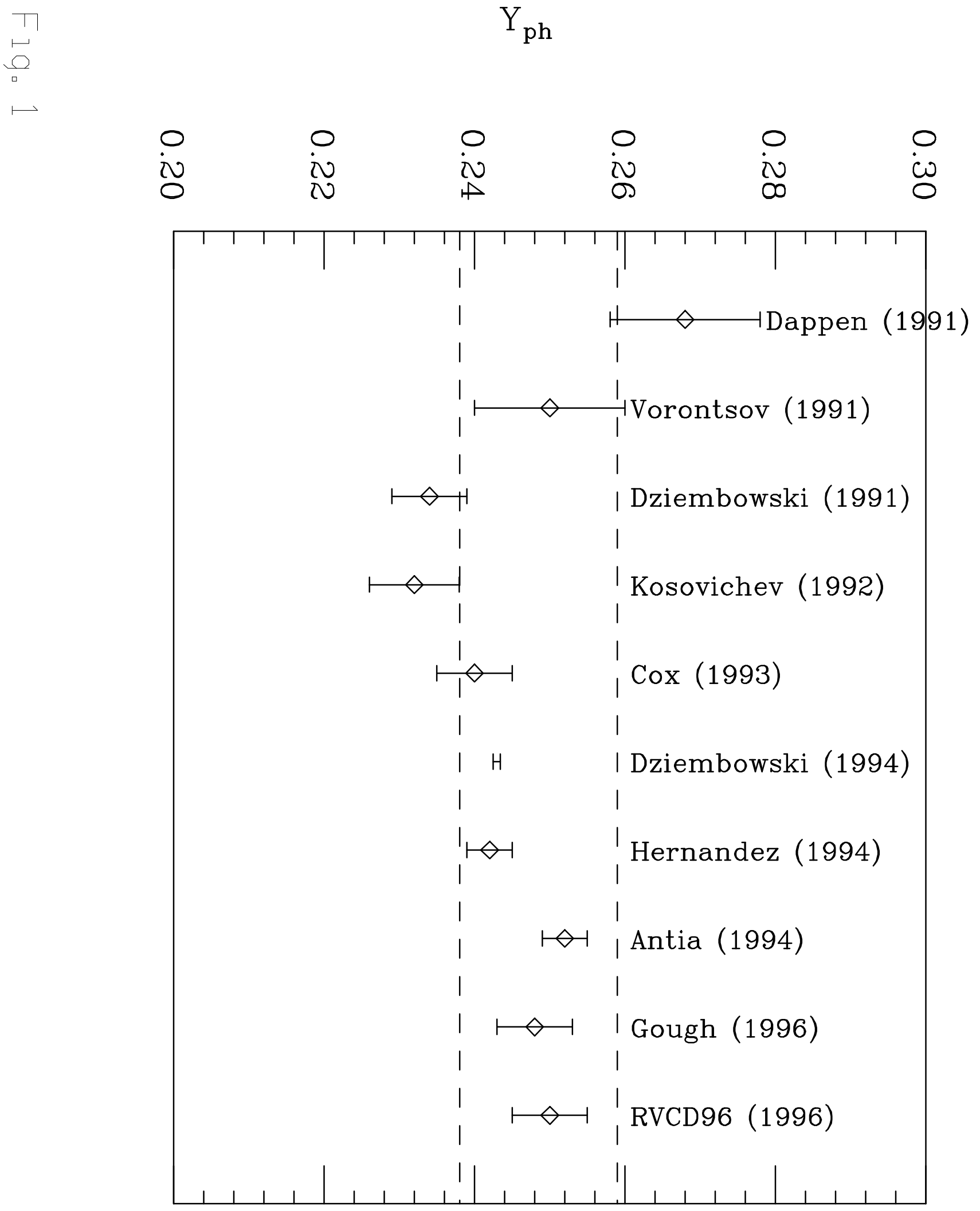


This figure "fig2.gif" is available in "gif" format from: http://arxiv.org/ps/astro-ph/9612053v1 


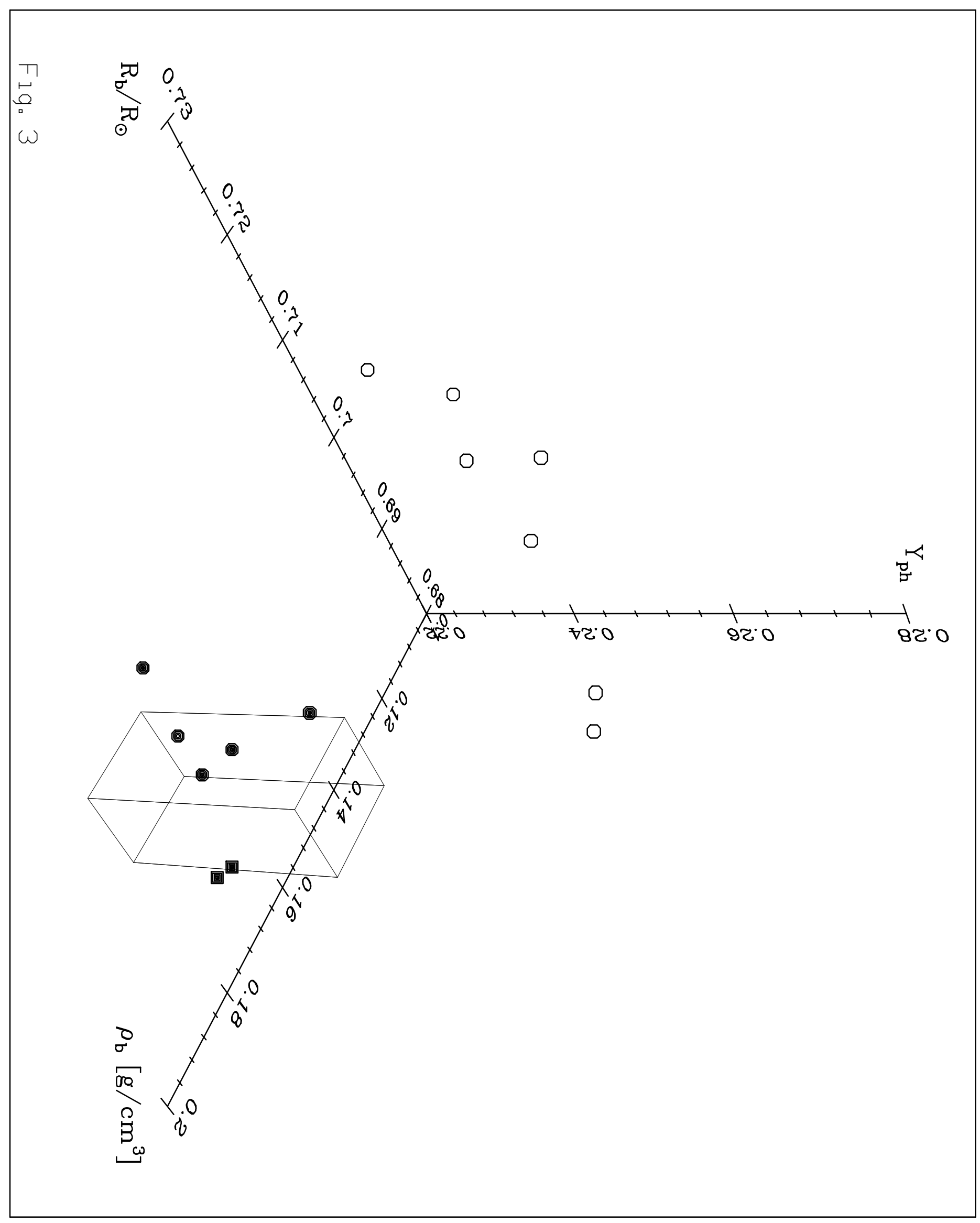


This figure "fig4.gif" is available in "gif" format from: http://arxiv.org/ps/astro-ph/9612053v1 


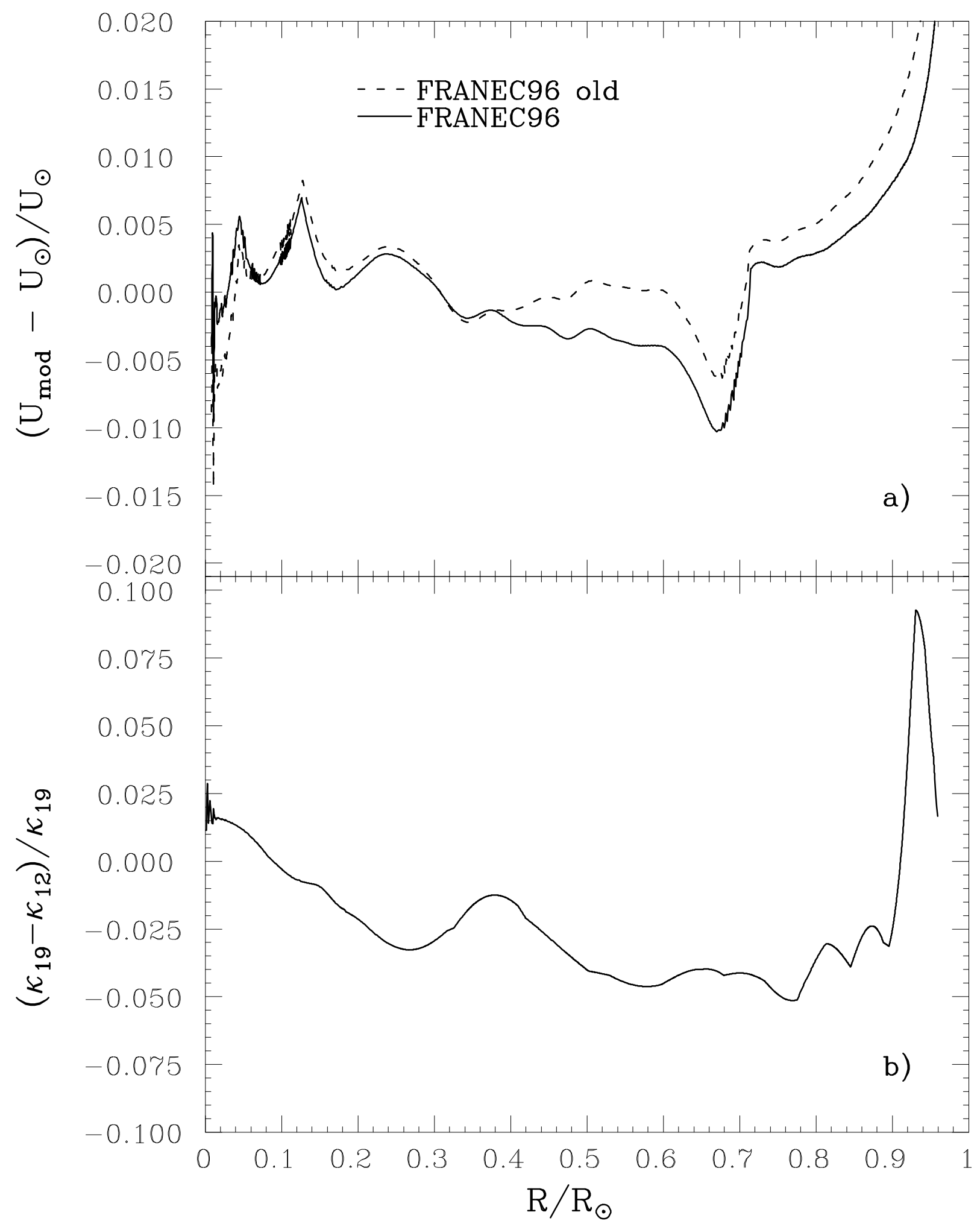

F19. 5 